\title{
A general dissipativity constraint for feedback system design, with emphasis on MPC
}

\author{
Tri $\operatorname{Tran}^{1}$, Jan Maciejowski ${ }^{2 *}$, and K-V. Ling ${ }^{3}$ \\ ${ }^{1}$ Curtin University, Bentley, Perth, Western Australia 6102, and \\ University of New South Wales, Sydney, NSW 2052, Australia; \\ ${ }^{2}$ Department of Engineering, University of Cambridge, Cambridge CB2 1PZ, United Kingdom, \\ and Energy Research Institute at Nanyang Technological University (ERI@N); \\ ${ }^{3}$ School of Electrical and Electronic Engineering, Nanyang Technological University, Block S2, 50 Nanyang Ave, \\ Singapore 639798.
}

\begin{abstract}
SUMMARY
A 'General Dissipativity Constraint' (GDC) is introduced to facilitate the design of stable feedback systems. A primary application is to MPC controllers when it is preferred to avoid the use of 'stabilising ingredients' such as terminal constraint sets or long prediction horizons. Some very general convergence results are proved under mild conditions. The use of quadratic functions, replacing GDC by 'Quadratic Dissipation Constraint' (QDC), is introduced to allow implementation using linear matrix inequalities. The use of QDC is illustrated for several scenarios: state feedback for a linear time-invariant system, MPC of a linear system, MPC of an input-affine system, and MPC with persistent disturbances. The stability that is guaranteed by GDC is weaker than Lyapunov stability, being 'Lagrange stability plus convergence'. Input-to-state stability is obtained if the control law is continuous in the state. An example involving an open-loop unstable helicopter illustrates the efficacy of the approach in practice. Copyright (c) 2018 John Wiley \& Sons, Ltd. Received: 12 Dec 2017. Revised: 2019.
\end{abstract}

KEY WORDS: Dissipativity, Feedback design, Linear matrix inequality, Model predictive control, Quadratic dissipativity constraint.

\section{INTRODUCTION}

Ensuring stability of a feedback system by constructing a Lyapunov function is conservative, in the sense that a specific Lyapunov function is only a sufficient condition for stability in general. Alternative procedures, such as ensuring the existence of a Lyapunov function in Model Predictive Control (MPC) by specifying a terminal constraint set, or a long prediction horizon, are also

\footnotetext{
* Submitted author: Prof. J.M. Maciejowski, Cambridge University Engineering Dept, Trumpington Street, Cambridge CB2 1PZ, UK - email: jmm@eng.cam.ac.uk

Contract/grant sponsor: The authors acknowledge support by the Singapore National Research Foundation (NRF) under its Campus for Research Excellence And Technological Enterprise (CREATE) programme, specifically the Cambridge Centre for Advanced Research and Education in Singapore (Cambridge CARES, http://www.cares.cam.ac.uk), project C4T.
} 
conservative, for the same reason. This paper proposes a relaxation of the usual property of a Lyapunov function, namely that it should never increase, together with a General Dissipativity Constraint (GDC) that holds along (closed-loop) solution trajectories. This combination guarantees boundedness of the state trajectory, and convergence to a point or a set, depending on whether persistent disturbances are present or not. Our development is inspired by dissipativity theory; it introduces a general 'supply function' and a 'storage function' which is the relaxed quasi-Lyapunov function.

Of course, if stability is obtained, then a conventional Lyapunov function also exists, so our development may appear to be redundant. The motivation is to facilitate constructive design of feedback control systems. The GDC allows one to search over a wider set of possible solutions within the domain of interest, which can be exploited in either off-line design, or when using online decision-making, as in MPC. All of our development is in a discrete-time setting, motivated by our use of GDC mostly in the context of MPC with constraints.

We have applied the GDC concept successfully in a number of application problems. In these applications we have specialised the supply and storage functions to be quadratic, and hence used the term 'Quadratic Dissipativity Constraint (QDC)' instead of 'GDC'. In [24] and [26] the concept was applied to decentralised MPC and decentralised static state-feedback of electrical power systems using reduced-order linearized (small-signal) models. In these works the QDC condition was translated into an additional quadratic constraint on the input vector. In [27] the GDC/QDC concept was applied to MPC control of a nonlinear input-affine system, by solving online a linear matrix inequality (LMI) for the coefficient matrices of a quadratic supply function which satisfied a QDC condition. Numerical simulation with a continuous stirred-tank reactor illustrated the result. In [28] a QDC condition was combined with an 'iterative feasibility' condition, and applied to the control of two open-loop unstable interconnected systems. It was demonstrated that an online choice of QDC coefficient matrices may be needed in this case (as opposed to a fixed choice, decided off-line). In [29] a QDC condition was applied in a 'multiplexed MPC' scheme for Automatic Generation Control (AGC) in a 4-area electrical power system.

The purpose of this paper is to establish, rigorously and clearly, the basic results underlying the use of GDC/QDC, without obscuring them by details of interconnected systems or complex applications.

The idea of having a dissipation-based constraint on the controller in a control design problem has appeared in previous work, such as [17, 3, 25, 13, 23]. In [3] a dissipativity constraint was applied to the closed loop in order to obtain satisfactory performance (by constraining the $L_{2}$ gain from a disturbance to a performance variable). A quadratic storage function was introduced, and the supply function depended on the magnitudes (only) of the disturbance and the performance variable. A (discrete-time) moving-horizon $H_{\infty}$ scheme was suggested as a practical implementation of this idea. The dissipativity constraint and disturbance specification were converted into LMIs, which were solved online to give a time-varying state-feedback control $u(k)=K(k) x(k)$. Feasibility was guaranteed if the disturbance was small enough, but heuristic schemes for coping with infeasibility were briefly considered. [17] introduced similar ideas into (continuous-time) nonlinear MPC with an infinite horizon and without constraints, but with dissipativity restricted to classical passivity. It was pointed out that the passive output need not be a real output of the plant. In [25] closed-loop stability of MPC in the presence of data errors, such as packet drops in the communication between 
controller and plant, was obtained by adding a "cumulative quadratic constraint" (a discrete-time version of an Integral Quadratic Constraint) to the usual MPC formulation. The plant was assumed to be dissipative with a quadratic supply function, and some off-line LMI computations were required to specify the additional constraint, which modified the optimisation problem to be solved online. [13] introduced parametrised ISS Lyapunov functions, and parametrised dissipation inequalities, to obtain robust stability of (discrete-time, nonlinear) MPC in the presence of additive disturbances. The parametrised Lyapunov functions were defined on the basis of robustly positive invariant sets, which allowed recursive feasibility results to be obtained. Quadratic and polyhedral supply functions were considered, while Lyapunov functions of general structure were allowed (although quadratic ones were mostly considered). In some ways [23] is the closest of these papers to our paper in concept, in that it enforces dissipative trajectories, which is a slightly weaker notion than the classical notion of dissipativity of a system. Again it imposes an additional dissipativity constraint on the usual MPC problem formulation. [23] considers quadratic supply functions, but the main departure from previous papers, and from this paper, is that the dissipativity constraint depends on predicted inputs and outputs (states) as well as on current and previous ones. Note that [23] requires open-loop dissipativity and a monotonic Lyapunov function. Neither of these are required by the development in this paper.

Most of the ideas in this paper have already appeared in at least one of the papers reviewed above, but not all together in any one of them. Shared ideas are that we impose a dissipativity condition as an additional constraint in the usual MPC problem formulation, that we make a lot of use of LMIs to perform specific computations associated with quadratic storage and supply functions, and we aim to do as much of this as possible off-line in each case considered, so as to minimise the online, real-time computational burden. The main innovation in our work is the use of very general storage and supply functions; the convergence results in section 3 do not require any specific structure - dissipation inequalities (4) and (8) are more general than any that appear in the papers reviewed above (except [23] in which the functions are more complicated than here) since the supply function $\xi$ only has to satisfy a summability condition (see (2), specialised later in Lemma 1 and Theorem 2, in particular (9) and (10).) In sections 4 and 5 we specialise to quadratic functions in order to be able to perform specific computations, but even then the $\xi$ term in (4) and (8) is relatively general. The price of this generality is that we obtain (only) asymptotic convergence results, and inputto-state stability is obtained in the cases with disturbance under a mild condition; features such as continuity in the initial conditions need to come from application-dependent features, while features such as recursive feasibility need additional constraints if they are to be enforced. The benefit of the generality is a less stringent condition to be satisfied, so that the real-time optimisation problem (in MPC) becomes easier.

The paper is structured as follows. The next section introduces some notation and our assumed system model. Section 3 then introduces a very general GDC, and presents two theorems which prove boundedness and convergence of the state trajectory of a system, together with a lemma which helps with practical implementation, and a corollary which significantly strengthens one of the convergence results to ISS stability. Section 4, the longest section in the paper, shows how to apply the very general results of section 3 in several situations: state feedback for a linear time-invariant system (section 4.1), MPC of a linear system (section 4.2), MPC of an input-affine system (section 4.3), and MPC with persistent disturbances (section 4.4). Section 5 then provides 
an extended discussion of the results. In particular, it points out that the GDC condition ensures Lagrange stability (boundedness) and convergence, but not Lyapunov stability.

However, Corollary 1 and Remark 3 in section 3 show that input-to-state stability is often obtained.) Section 6 contains a simplified application example, and section 7 concludes the paper. The proofs of the two main theorems of section 3 are located in the appendices.

\section{PRELIMINARIES}

\subsection{Notation}

Upper and lower case letters denote matrices and column vectors, respectively. $(.)^{T}$ denotes the transpose of a vector or a matrix. $\|u\|$ is the (Euclidean) 2 -norm of vector $u$. The (discrete) time index is denoted by $k \in \mathbb{Z}$. Functions of classes $\mathcal{K}, \mathcal{K}_{\infty}, \mathcal{L}$ and $\mathcal{K} L$ are defined as follows: A function $\alpha: \mathbb{R}_{0}^{+} \rightarrow \mathbb{R}_{0}^{+}$is of class $\mathcal{K}$ if it is continuous, strictly increasing and $\alpha(0)=0$, and is of class $\mathcal{K}_{\infty}$ if, in addition, it is unbounded. A function $\alpha: \mathbb{R}_{0}^{+} \rightarrow \mathbb{R}_{0}^{+}$is of class $\mathcal{L}$ if it is decreasing and $\lim _{t \rightarrow \infty} \alpha(t)=0$. A function $\alpha: \mathbb{R}_{0}^{+} \times \mathbb{R}_{0}^{+} \rightarrow \mathbb{R}_{0}^{+}$is of class $\mathcal{K} \mathcal{L}$ if it is of class $\mathcal{K}_{\infty}$ in the first argument and of class $\mathcal{L}$ in the second argument. For real $s,\lfloor s\rfloor$ denotes the largest integer smaller than or equal to $s$.

\subsection{System model}

Consider a discrete-time system $\mathscr{S}$ of the form:

$$
\mathscr{S}: x(k+1)=f(x(k), u(k))+d(k)
$$

where $x \in \mathbb{X} \subseteq \mathbb{R}^{n}, u \in \mathbb{U} \subseteq \mathbb{R}^{m}$ are the state and control vectors, respectively. We assume, without loss of generality, that $0 \in \mathbb{X}, 0 \in \mathbb{U}$, and $f(0,0)=0 . d(k) \in \mathbb{D} \subset \mathbb{R}^{n}$ represents an unknown but bounded persistent input disturbance, with $\|d(k)\|^{2} \leq \theta<\infty$. We assume sufficient regularity of $f$ to ensure existence and uniqueness of solutions.

\section{SOME CONVERGENCE RESULTS}

\section{Theorem 1}

Consider the system $\mathscr{S}$ with $\theta=0$ (i.e. without disturbance $d$ ) and some $\tau$ such that $0<\tau<1$. Suppose there are two $\mathcal{K}_{\infty}$ functions $\underline{\alpha}(\cdot), \bar{\alpha}(\cdot)$, a real-valued non-negative function $V(k, x)$ with finite $V(0, x(0))$, a control sequence $\{u(k)\}$, a function $\xi(k, x(k), u(k))$, such that

$$
\sum_{k=0}^{\kappa}|\xi(k, x(k), u(k))| \leq \Xi \text { for all } \kappa>0 \text { and for some } \Xi<\infty
$$

( $\Xi$ independent of $\kappa$ ), and the following holds for all $k>0$ :

$$
\underline{\alpha}(\|x(k)\|) \leq V(k, x(k)) \leq \bar{\alpha}(\|x(k)\|),
$$


and

$$
V(k+1, x(k+1))-\tau V(k, x(k)) \leq|\xi(k, x(k), u(k))| .
$$

Then $x(k)$ remains bounded and $\|x(k)\| \rightarrow 0$ as $k \rightarrow \infty$.

\section{Proof}

In Appendix A.

Remark 1 1. We avoid imposing any continuity conditions on $V$, because the primary intended application of these results is to MPC. MPC control laws are in general discontinuous, even if the system model is continuous, because of constraints.

2. In standard Lyapunov theory the right-hand side of the inequality in (4) is usually 0 . The generalisation here allows some reduction of the conservativeness inherent in this sufficient condition, and is typical in dissipativity theory.

3. The function $|\xi(\cdot, \cdot, \cdot)|$ plays the role here of a supply function in the theory of dissipative systems. The summability condition (2) resembles the condition $-\int s(t) d t \leq K$ in [30], and (4) resembles a dissipativity condition.

In applications condition (2) needs to be enforced, or at least checked. In general this can only be done by imposing further conditions on the function $\xi$. A particular sufficient condition is given in the following Lemma:

\section{Lemma 1}

If

$$
|\xi(k, x(k), u(k))| \leq \alpha(|\xi(0, x(0), u(0))|, k)
$$

where $\alpha(\cdot, \cdot)$ is some $\mathcal{K} \mathcal{L}$ function such that $\alpha(s, k+1) \leq \gamma \alpha(s, k)$ for some positive $\gamma<1$, then condition (2) holds.

Proof

$$
\begin{aligned}
\sum_{k=0}^{\infty}|\xi(k, x(k), u(k))| \leq \sum_{k=0}^{\infty} \alpha(\mid \xi & (0, x(0), u(0)) \mid, k) \\
\leq \sum_{k=0}^{\infty} \gamma^{k} \alpha(|\xi(0, x(0), u(0))|, 0) & =\frac{\alpha(|\xi(0, x(0), u(0))|, 0)}{1-\gamma}
\end{aligned}
$$

\section{Remark 2}

The condition in Lemma 1 is certainly not necessary for such a theorem to hold. It is only necessary for $\alpha$ to decrease sufficiently quickly with $k$ so that a summability condition such as (2) holds.

We now give a theorem that applies when disturbances are present, namely without assuming that $\theta=0$. It uses a condition similar to that which appears in Lemma 1.

\section{Theorem 2}

Consider the system $\mathscr{S}$ and some $\tau$ such that $0<\tau<1$. Suppose there are two $\mathcal{K}_{\infty}$ functions $\underline{\alpha}(\cdot)$, $\bar{\alpha}(\cdot)$, a real-valued non-negative function $V(k, x)$ with finite $V(0, x(0))$, a control sequence $\{u(k)\}$, 
and a function $\xi(k, x(k), u(k))$, such that the following hold for all $k>0$ :

$$
\underline{\alpha}(\|x(k)\|) \leq V(k, x(k)) \leq \bar{\alpha}(\|x(k)\|),
$$

and

$$
V(k+1, x(k+1))-\tau V(k, x(k)) \leq|\xi(k, x(k), u(k))|+\sigma_{1} d(k)^{T} d(k)
$$

for some $\sigma_{1}>0$, and

$$
|\xi(k, x(k), u(k))| \leq \alpha(|\xi(0, x(0), u(0))|, k)+\sigma_{2} \theta
$$

for some $\sigma_{2}>0$, where $\alpha(\cdot, \cdot)$ is some $\mathcal{K} \mathcal{L}$ function such that

$$
\alpha(s, k+1) \leq \gamma \alpha(s, k)
$$

for some positive $\gamma<1$. Then

$$
\|x(k)\| \leq \alpha_{0}(\|x(0)\|, k)+\alpha_{1}(|\xi(0, x(0), u(0))|, k)+\beta(\theta)
$$

where $\alpha_{0}$ and $\alpha_{1}$ are $\mathcal{K} \mathcal{L}$ functions and $\beta$ is a $\mathcal{K}$ function.

\section{Proof}

In Appendix B.

Corollary 1

If the conditions of Theorem 2 hold, and in addition

$$
|\xi(0, x(0), u(0))| \leq \rho(\|x(0)\|)
$$

where $\rho$ is a $\mathcal{K}$ function, then

$$
\|x(k)\| \leq \alpha_{2}(\|x(0)\|, k)+\beta(\theta)
$$

where $\alpha_{2}$ is a $\mathcal{K} \mathcal{L}$ function.

Proof

Since the set of $\mathcal{K}$ functions is closed under composition, and the set of $\mathcal{K} \mathcal{L}$ functions is closed under addition, we define

$$
\alpha_{2}(s, k)=\alpha_{0}(s, k)+\alpha_{1}(\rho(s), k)
$$

and the result follows from (11).

\section{Remark 3}

Property (13) defines input-to-state stability, if proved for a suitable set of initial conditions. Note that (12) holds whenever the control law is a continuous function of the state, if $\xi(k, x, u)$ is continuous in $x$ and $u$.

\section{Remark 4}

Conditions such as those that appear in Theorems 1 and 2 are clearly not necessary for convergence

Copyright (c) 2018 John Wiley \& Sons, Ltd. Received: 12 Dec 2017. Revised: 2019.Int. J. Robust. Nonlinear Control (2018), 
results to hold. Standard converse Lyapunov results show that the introduction of the supply function $\xi$ is not necessary. The motivation for introducing these conditions is to provide a wider set of sufficient conditions, in order to facilitate the search for stabilisiing controls.

\section{Remark 5}

We call conditions such as (4) and (8), together with the summability condition (2) (possibly obtained by conditions such as those in Lemma 1), General Dissipativity Constraints, abbreviated as $G D C$. Usually the storage function $V$ and the supply function $\xi$ are chosen to be quadratic, in which case we also use the term Quadratic Dissipativity Constraints, abbreviated as QDC.

\section{APPLICATION OF GDC/QDC}

The results presented in the previous section relate to the behaviour of individual trajectories of a controlled dynamic system. In order to use them to obtain stability results we will specialise both $V$ and $\xi$ to be quadratic functions, we will consider mostly linear models, and we will make use of linear matrix inequalities (LMIs) to enforce the required conditions. At the end of each subsection of this section we summarise by giving a Proposition and a Summary procedure in the form of a pseudo-algorithm.

\subsection{Application to linear state-feedback design}

As a first illustration we shall apply the results of section 3 to (offline) design of a state-feedback scheme. For clarity we consider a linear model without disturbances, so that (1) becomes

$$
x(k+1)=A x(k)+B u(k)
$$

and we look for a state-feedback matrix $K$, namely $u(k)=K x(k)$, such that $\|x(k)\| \rightarrow 0$ for all $x(0)$. (In this case $\mathbb{X}=\mathbb{R}^{n}$.) We assume that the pair $(A, B)$ is stabilisable.

We take $V(x)=x^{T} P x$ for some (as yet undetermined) $P=P^{T}>0$. We also take $\xi$ to be (in this case time-invariant):

$$
\xi(k, x, u)=\xi(x, u)=x^{T} Q x+2 x^{T} S u+u^{T} R u .
$$

In this case condition (4) becomes

$$
x(k)^{T}\left[(A+B K)^{T} P(A+B K)-\tau P\right] x(k) \leq x(k)^{T} M x(k)
$$

where we define

$$
M=Q+S K+K^{T} S^{T}+K^{T} R K
$$

This is certainly satisfied if

$$
(A+B K)^{T} P(A+B K)-\tau P \leq M
$$


which, by using the Schur complement, is the same as the condition

$$
\left[\begin{array}{cc}
P^{-1} & A+B K \\
(A+B K)^{T} & \tau P+M
\end{array}\right] \geq 0
$$

Now inequality (20) is not an LMI, but it can be converted into an LMI by introducing the new variables $X=K P^{-1}, Y=M P^{-1}$ and $W=P^{-1} Y$ : pre- and post-multiplying (20) by $\operatorname{diag}\left[I, P^{-1}\right]$, and making the inequality strict, gives

$$
\begin{aligned}
& {\left[\begin{array}{cc}
P^{-1} & A P^{-1}+B K P^{-1} \\
\left(A P^{-1}+B K P^{-1}\right)^{T} & \tau P^{-1}+P^{-1} M P^{-1}
\end{array}\right]=} \\
& {\left[\begin{array}{cc}
P^{-1} & A P^{-1}+B X \\
\left(A P^{-1}+B X\right)^{T} & \tau P^{-1}+W
\end{array}\right]>0}
\end{aligned}
$$

which is an LMI in the variables $P^{-1}, W$ and $X-$ from which $Y$ can be recovered as $Y=P W$.

In order to meet the condition of Lemma 1 we impose the condition

$$
0 \leq \xi(x(k+1), u(k+1)) \leq \gamma \xi(x(k), u(k))
$$

for some $0<\gamma<1$, namely

$$
0 \leq x(k)^{T}(A+B K)^{T} M(A+B K) x(k) \leq \gamma x(k)^{T} M x(k) .
$$

This is certainly satisfied if

$$
\gamma M-(A+B K)^{T} M(A+B K) \geq 0 \quad \text { and } \quad M>0,
$$

which, again by using the Schur complement, is implied by the condition

$$
\left[\begin{array}{cc}
M^{-1} & A+B K \\
(A+B K)^{T} & \gamma M
\end{array}\right]>0 .
$$

This is a nonlinear matrix inequality, because of the presence of both $M$ and $M^{-1}$. We proceed as follows. We take solutions $X_{0}$ and $Y_{0}$ to (21), and we look for a new value of $P$ which satisfies both (21) and (25), with $X$ and $Y$ fixed at the values $X_{0}$ and $Y_{0}$ :

$$
\begin{aligned}
& {\left[\begin{array}{cc}
P^{-1} & A P^{-1}+B X_{0} \\
\left(A P^{-1}+B X_{0}\right)^{T} & \tau P^{-1}+P^{-1} Y_{0}
\end{array}\right]>0} \\
& {\left[\begin{array}{cc}
P^{-1} Y_{0}^{-1} & A P^{-1}+B X_{0} \\
\left(A P^{-1}+B X_{0}\right)^{T} & \gamma P^{-1} Y_{0}
\end{array}\right]>0}
\end{aligned}
$$

This is an LMI in $P^{-1}$. When this is solved, we recover

$$
K=X_{0} P \quad \text { and } \quad M=Y_{0} P
$$


Note that we do not need to recover the matrices $Q, R, S$ from $M$. In the closed loop we have $\xi(x(k), u(k))=x(k)^{T} M x(k)$ and this is guaranteed to be nonnegative since $M>0-$ so we do not have to consider the absolute value in (4). (Other procedures for solving LMIs (20) and (25) are possible; see, for example, [19].)

Clearly, using this machinery for the basic state-feedback problem is unnecessarily complicated compared with other algorithms. However, the same approach can be used for the design of decentralised state-feedback in case the system (15) is an interconnection of subsystems [24].

If using standard Lyapunov theory, the search for a stabilising $K$ would involve finding $K$ and $P=P^{T}>0$ such that $(A+B K)^{T} P(A+B K)-P<0$, which is equivalent to

$$
\left[\begin{array}{cc}
P & (A+B K)^{T} \\
(A+B K) & P^{-1}
\end{array}\right]>0 .
$$

Comparing (29) with (20) we see that (20) is easier to satisfy, since there are additional degrees of freedom introduced by the presence of the matrix $M$, despite the structural restrictions visible in (28).

Note that in this application the bounding $\mathcal{K}$-functions $\underline{\alpha}$ and $\bar{\alpha}$ (in Theorem 1) can be taken to be $\underline{\alpha}(s)=s^{2} \lambda_{\min }(P)$ and $\bar{\alpha}(s)=s^{2} \lambda_{\max }(P)$, where $\lambda_{\min }(P)$ and $\lambda_{\max }(P)$ are the smallest and largest eigenvalues of $P$, respectively. The bounding $\mathcal{K} \mathcal{L}$-function $\alpha$ (in Lemma 1 ) can be taken to be $\alpha(s, k)=\gamma^{k} s$. $\tau$ and $\gamma$ are parameters of the design procedure; both of them should be chosen to be in the interval $(0,1)$. Reducing $\tau$ forces $V(\|x\|)$ to reduce more quickly, or at least to grow less quickly (since (4) allows $V$ to increase to some extent), and thus leads to faster convergence of $x$ to 0 . On the other hand, reducing $\tau$ makes it more difficult to satisfy (20) (or (26)), and a suitable trade-off has to be found for each particular system. Similar considerations apply to the choice of $\gamma$, although its effect on the rate of convergence of $x$ to 0 is less direct. Reducing $\gamma$ tends to increase the convergence rate, but makes it more difficult to satisfy (25) (or (27)).

The following proposition and procedure summarize the development in this sub-section:

\section{Proposition 1}

Consider the system (15), and some $0<\tau<1$ and $0<\gamma<1$. Let $P_{0} \succ 0, W_{0}$, and $X_{0}$ be a solution to (21), set $Y_{0}=P_{0} W_{0}$, and let $P \succ 0$ be a solution to (26) and (27). Set $K=X_{0} P$. Then the state feedback system (15) with $u(k)=K x(k)$ is dissipative with $V(x)=x^{T} P x$ and $\xi(x, u)=x^{T} Q x+2 x^{T} S u+u^{T} R u$, and the QDC $0 \leq \xi(x(k+1), u(k+1)) \leq \gamma \xi(x(k), u(k))$ is always satisfied. The closed-loop system is asymptotically attractive.

\section{Summary procedure 1}

\section{Offline:}

1. Choose $\tau$ and $\gamma$.

2. Solve (21) for $P_{0}, W_{0}, X_{0}$. Set $Y_{0}=P_{0} W_{0}$.

3. Solve (26) and (27) for $P$.

4. Set $K=X_{0} P$ (and $M=Y_{0} P$ if wanted). 


\section{Online:}

1. $u(k)=K x(k)$

\subsection{Application to MPC with linear system}

In MPC the control signal is decided by solving, at time $k$, an optimisation problem of the form

$$
\min _{\mathbf{u}} \sum_{i=0}^{N} \ell\left(x_{i}, u_{i}\right)+F\left(x_{N+1}\right) \quad \text { subject to } \quad x_{i} \in \mathbb{X}, u_{i} \in \mathbb{U}
$$

where $x_{i+1}=f\left(x_{i}, u_{i}\right), x_{0}=x(k), \mathbf{u}=\left\{u_{0}, \ldots, u_{N}\right\}, \ell(\cdot, \cdot)$ is some 'stage cost', and $F(\cdot)$ is a 'terminal cost'. Note that it is assumed that $x(k)$ has been measured. $\ell$ and $F$ have suitable properties (such as positive-definiteness etc), and $F$ is often omitted. If $\mathbf{u}^{*}$ denotes the optimal solution to this problem then the control signal applied at time $k$ is $u(k)=u_{0}^{*}$. This process is repeated at the next step, with $x_{0}$ being reset to the next measured state $x(k+1)$. The sets $\mathbb{X}$ and $\mathbb{U}$ are implied by problem-dependent constraints. Frequently a 'terminal constraint' of the form $x_{N+1} \in \mathbb{T}$ is added to the problem, in order to ensure properties such as closed-loop stability and recursive feasibility, but the addition of constraints inevitably makes the optimisation problem more difficult to solve and increases the risk of hitting an infeasible problem (at $k=0$ ). If $f(\cdot, \cdot)$ is linear, $\ell(\cdot, \cdot)$ is quadratic (and non-negative), and all constraints are linear in $x$ and $u$, then minimisation (30) is a convex quadratic program (QP). For further details of MPC see [14, 18].

In the context of MPC, the QDC condition is translated into additional quadratic constraints on the input $u(k)$. These are used instead of a terminal constraint, in order to increase the likelihood of avoiding infeasibility. In the simplest case we choose $V(x)=x^{T} P x$, as before, for some $P=P^{T}>0$, and $\xi(x, u)=x^{T} Q x+2 x^{T} S u+u^{T} R u$. Using the system model (15), condition (4) becomes

$$
\begin{aligned}
u(k)^{T}\left[B^{T} P B-R\right] u(k)+2 u(k)^{T}\left[B^{T} P A-S^{T}\right] x(k)+ & \\
& x(k)^{T}\left[A^{T} P A-\tau P-Q\right] x(k) \leq 0
\end{aligned}
$$

To satisfy the condition of Lemma 1 we impose $\xi(x, u)>0$ and

$$
\xi(x(k), u(k)) \leq \gamma \xi(x(k-1), u(k-1))
$$

for some $0<\gamma<1$, which can be rewritten as

$$
\begin{aligned}
& u(k)^{T} R u(k)+2 u(k)^{T} S^{T} x(k) \leq \\
& \gamma\left[x(k-1)^{T}, u(k-1)^{T}\right]\left[\begin{array}{cc}
Q & S \\
S^{T} & R
\end{array}\right]\left[\begin{array}{l}
x(k-1) \\
u(k-1)
\end{array}\right]-x(k)^{T} Q x(k)
\end{aligned}
$$

and

$$
\left[\begin{array}{cc}
Q & S \\
S^{T} & R
\end{array}\right]>0
$$

Copyright (C) 2018 John Wiley \& Sons, Ltd. Received: 12 Dec 2017. Revised: 2019.Int. J. Robust. Nonlinear Control (2018), 
Note that $u(k)$ is the only unknown in (31) and (33). These two inequalities are quadratic constraints on $u(k)$, which therefore turn the minimisation problem (30) into a quadraticallyconstrained quadratic program instead of the more usual linearly-constrained quadratic program. Such problems are NP-hard in general, but if certain positive-definiteness conditions hold then they are convex, and can be solved using semi-definite programming [2].

In applications we have avoided imposing constraint (31) online, by solving the following LMI off-line:

$$
\left[\begin{array}{cc}
A^{T} P A-\tau P-Q & A^{T} P B-S \\
B^{T} P A-S^{T} & B^{T} P B-R
\end{array}\right] \leq 0
$$

If this is satisfied then (31) holds for every pair $x(k), u(k)$, and only (33) remains to be imposed as an online constraint. Furthermore this is a convex constraint, since $R>0$. The question of how to choose the matrices $P, Q, R, S$ remains. One procedure, which we have found to be effective, is to solve the problem

$$
\min _{P, Q, R, S} z^{T} Q z \quad \text { subject to (34) and (35) holding, and } P>0
$$

for some $z \in \mathbb{R}^{n}$. $z$ can be chosen to be a 'typical' value of $x(k)$, or a typical initial condition, or a 'max-min' problem can be solved for a finite set of $z$ 's. The motivation for this is that it is an attempt to maximise the right-hand side of (33) and thus to make that constraint more easily satisfiable. It is also possible to choose $P$ to be the solution to the algebraic Riccati equation associated with the $\operatorname{cost} \ell(x, u)$ (if it is quadratic and the model is linear), and to minimise (36) over $Q, R, S$ only. In this case it would be standard (but not necessary) to choose $F(x)=x^{T} P x$, so that the storage function is the same as the terminal cost.

In addition to LMIs (34) and (35), we can add a third LMI for determining $Q, R, S$ :

$$
\left[\begin{array}{cc}
A^{T} Q A-\gamma Q & A^{T} Q B-\gamma S \\
B^{T} Q A-\gamma S^{T} & B^{T} Q B-\gamma R
\end{array}\right] \leq 0
$$

If this holds then the right-hand side of (33) is nonnegative, and consequently (33) is always feasible, since $u(k)=0$ satisfies the inequality. In this case, if there are only input constraints $u_{i} \in \mathbb{U}$, but no state constraints, then the MPC problem (with the QDC constraint (33)) is always feasible, providing that $0 \in \mathbb{U}$. Note, however, that (37) can hold only if the open-loop system is (marginally) stable, and can hold with strict inequality only if the open-loop system is asymptotically stable, since it requires $A^{T} Q A-\gamma Q \leq 0$, while $Q>0$ (from (34)).

Since the storage function $V$ and the supply function $\xi$ are constant in this application, we can again choose $\underline{\alpha}(s)=s^{2} \lambda_{\min }(P)$ and $\bar{\alpha}(s)=s^{2} \lambda_{\max }(P)$ (in Theorem 1), and $\alpha(s, k)=\gamma^{k} s$ (in Lemma 1).

\section{Remark 6}

By following the procedure outlined in this section condition (4) of Theorem 1 is enforced for all possible inputs, whereas condition (2) is enforced 'along the trajectory' by choosing a suitable $u(k)$ at time $k$. 
Before leaving this section, we draw attention to a modification of (33) which is often necessary in practice. (33) can be rewritten as

$$
\begin{aligned}
{\left[u(k)-R^{-1} S^{T} x(k)\right]^{T} R\left[u(k)-R^{-1} S^{T} x(k)\right] \leq } \\
\gamma\left[x(k-1)^{T}, u(k-1)^{T}\right]\left[\begin{array}{cc}
Q & S \\
S^{T} & R
\end{array}\right]\left[\begin{array}{c}
x(k-1) \\
u(k-1)
\end{array}\right] \\
-x(k)^{T}\left[Q+S R^{-1} S^{T}\right] x(k)
\end{aligned}
$$

which shows that $u(k)$ must lie within an ellipsoid centred on $R^{-1} S^{T} x(k)$, whose principal semiaxes align with the eigenvectors of $R$, and whose volume is proportional to the right-hand side of the inequality. If this right-hand side is negative, then this inequality cannot be satisfied by any $u(k)$.

Note that one circumstance in which infeasibility due to this cause is likely to occur is when $u(k-1)$ is close to 0 but $x(k-1)$ is not close to 0 , and the sampling interval is small, as then $x(k) \approx x(k-1)$; the right-hand side can then become close to $-x(k)^{T} S R^{-1} S^{T} x(k)$ if $\gamma$ is close to 1 - which is usually the case. We find in some cases that this infeasibility occasionally occurs. When it does, we temporarily add a small positive constant to the right-hand side to restore feasibility. See also (55) in section 4.4.1, where a similar term is added (permanently) when disturbances are present - which is always the case, in practice.

The following proposition and procedure summarize the development in this sub-section:

\section{Proposition 2}

Consider the system (15) with constraint sets $\mathbb{X}$ and $\mathbb{U}$, the MPC problem (30), and some $0<\tau<1$ and $0<\gamma<1$. Let $P \succ 0, Q, R$, and $S$ be a solution to (36). Suppose that the right hand side of (33) is positive for $x(k), x(k-1) \in \mathbb{X} \backslash\{0\}$ and $u(k-1) \in \mathbb{U} \backslash\{0\}$, for all $k>0$. Let the control sequence $\mathbf{u}(x(k))=\left\{u_{0}, \ldots, u_{N}\right\}$ be a solution to the MPC optimization (30) subject to (33) at time step $k$. Then the state feedback system (15) with $u(x(k))=u_{0} \in \mathbf{u}(x(k))$ is dissipative with $V(x)=x^{T} P x$ and $\xi(x, u)=x^{T} Q x+2 x^{T} S u+u^{T} R u$, and the QDC $0 \leq \xi(x(k), u(k)) \leq$ $\gamma \xi(x(k-1), u(k-1))$ is satisfied for all $k>0$. The closed-loop system is asymptotically attractive.

\section{Summary procedure 2}

\section{Offline:}

1. Choose $\tau$ and $\gamma$. Choose $z \in \mathbb{R}^{n}$.

2. Solve (36) for $P, Q, R, S$ or fix $P>0$ (eg as solution of ARE) and solve (36) for $Q, R, S$.

\section{Online:}

1. Solve (30) subject to (33) (and optionally (37) if the system is open-loop stable).

2. If infeasible, add small constant to right-hand side of (33) and repeat.

3. Set $u(k)=u_{0}$, where $\mathbf{u}=\left\{u_{0}, \ldots, u_{N}\right\}$ is the obtained solution. 


\subsection{Application to MPC with input-affine system}

Now suppose that we wish to control an input-affine system:

$$
x(k+1)=f(x(k))+G(x(k)) u(k)
$$

For notational convenience we will write $f_{k}=f(x(k))$ and $G_{k}=G(x(k))$. In this case we take $V(k, x)=x^{T} P_{k} x$ for some $P_{k}=P_{k}^{T}>0$. Also we define a 'virtual output' $y(k)=\left[x(k)^{T}, f_{k}^{T}\right]^{T}$ and

$$
\xi(k, x(k), u(k))=y(k)^{T} Q_{k} y(k)+2 y(k)^{T} S_{k} u(k)+u(k)^{T} R_{k} u(k)
$$

In this case we shall be choosing the matrices $P_{k}, Q_{k}, R_{k}, S_{k}$ online, depending on the latest state measurement $x(k)$. Note that the results in section 3 allow the functions $V$ and $\xi$ to depend on $k$, and we exploit this possibility in this subsection (also later in subsection 4.4.2).

Using the system model (39), condition (4) becomes

$$
\begin{array}{r}
{\left[f_{k}^{T}+u(k)^{T} G_{k}^{T}\right] P_{k+1}\left[f_{k}+G_{k} u(k)\right]-\tau x(k)^{T} P_{k} x(k) \leq} \\
y(k)^{T} Q_{k} y(k)+2 y(k)^{T} S_{k} u(k)+u(k)^{T} R_{k} u(k)
\end{array}
$$

which can be rewritten as

$$
\left[u(k)^{T} \quad 1\right] M_{k}\left[\begin{array}{c}
u(k) \\
1
\end{array}\right] \leq 0
$$

where

$$
M_{k}=\left[\begin{array}{cc}
G_{k}^{T} P_{k+1} G_{k}-R_{k} & G_{k}^{T} P_{k+1} f_{k}-S_{k}^{T} y(k) \\
f_{k}^{T} P_{k+1} G_{k}-y(k)^{T} S_{k} & f_{k}^{T} P_{k+1} f_{k}-\tau x(k)^{T} P_{k} x(k)-y(k)^{T} Q_{k} y(k)
\end{array}\right]
$$

and (41) is satisfied for all $u(k)$ if

$$
M_{k} \leq 0
$$

which is an LMI in $P_{k+1}, P_{k}, Q_{k}, R_{k}, S_{k}$, or in $P_{k+1}, Q_{k}, R_{k}, S_{k}$ if $P_{k}$ is taken to be the value found at the previous time step.

Once these matrices have been determined, satisfying the condition of Lemma 1 can again be ensured by imposing $\xi(k, x, u)>0$ and

$$
\xi(k, x(k), u(k)) \leq \gamma \xi(k-1, x(k-1), u(k-1))
$$

for some $0<\gamma<1$, which in this case becomes

$$
\begin{aligned}
& u(k)^{T} R_{k} u(k)+2 u(k)^{T} S_{k} x(k) \leq \\
& \gamma\left[y(k-1)^{T}, u(k-1)^{T}\right]\left[\begin{array}{cc}
Q_{k-1} & S_{k-1} \\
S_{k-1}^{T} & R_{k-1}
\end{array}\right]\left[\begin{array}{c}
y(k-1) \\
u(k-1)
\end{array}\right]-y(k)^{T} Q_{k} y(k)
\end{aligned}
$$

and

$$
\left[\begin{array}{ll}
Q_{k} & S_{k} \\
S_{k}^{T} & R_{k}
\end{array}\right]>0
$$


As in the previous section, the required matrices can be determined by solving the problem

$$
\min _{P_{k+1}, P_{k}, Q_{k}, R_{k}, S_{k}} y(k)^{T} Q_{k} y(k) \quad \text { subject to (44) and (47) holding, }
$$

but now this has to be solved online. Minimising $y(k)^{T} Q_{k} y(k)$ maximises the right-hand side of (46), which should make it easier to satisfy that constraint - which is again added as a constraint in the MPC computation. The values of $Q_{k-1}, R_{k-1}, S_{k-1}$, which are needed in (46), should be taken as the values obtained for $Q_{k}, R_{k}, S_{k}$ at the previous time step.

In this case, linking the algorithm given above to Theorem 1 and Lemma 1 is a little more complicated than in the two previous cases, since the parameters of both the storage function $V$ and the supply function $\xi$ may change at each step. Condition (3) is met by introducing two constants $c_{1}$ and $c_{2}$, with $0<c_{1}<c_{2}$, and imposing the additional constraints

$$
P_{k+1}-c_{1} I \geq 0 \quad \text { and } \quad c_{2} I-P_{k+1} \geq 0
$$

so that we have $\underline{\alpha}(\|x\|)=c_{1}\|x\|^{2}$ and $\bar{\alpha}(\|x\|)=c_{2}\|x\|^{2}$. (This has also been done in [13], for example.) To demonstrate compliance with Lemma 1 we can take $\alpha(s, k)=\gamma^{k} s$, as before.

Note that it may not be necessary to solve (48) at every step. If $x(k)$, and hence $f_{k}$, does not change much at each step then (44) may continue to be satisfied with the existing values of the matrices even when current values of $x(k)$ and $f_{k}$ are inserted in $M_{k}$. It is therefore advisable to check whether (44) holds at each step (which is computationally cheap) and to solve (48) only when it does not hold. This is likely to be particularly advantageous when regulating about a constant set-point, in the absence of large transients or disturbances.

The following proposition and procedure summarize the development in this sub-section:

\section{Proposition 3}

Consider the system (39) with constraint sets $\mathbb{X}$ and $\mathbb{U}$, the MPC problem (30), and some $0<\tau<1$ and $0<\gamma<1$. Let $P_{k+1} \succ 0, P_{k} \succ 0, Q_{k}, R_{k}$, and $S_{k}$ be a solution to (48), subject to (49) with current values of $x(k), y(k), f_{k}, G_{k}$, at time step $k$. Suppose that the right hand side of (46) is positive for $x(k), x(k-1) \in \mathbb{X} \backslash\{0\}$ and $u(k-1) \in \mathbb{U} \backslash\{0\}$, for all $k>0$, where $Q_{k-1}, R_{k-1}, S_{k-1}$ are taken as the values obtained for $Q_{k}, R_{k}, S_{k}$ at the previous time step. Let the control sequence $\mathbf{u}(x(k))=\left\{u_{0}, \ldots, u_{N}\right\}$ be a solution to the MPC optimization (30) subject to (46) at time step $k>0$. Then the state feedback system (39) with $u(x(k))=u_{0} \in \mathbf{u}(x(k))$ is dissipative with $V(k, x)=x^{T} P_{k} x$ and $\xi(k, x, u)=y T Q_{k} y+2 y^{T} S_{k} u+u^{T} R_{k} u$, and the (time-dependent) QDC $0 \leq \xi(k, x(k), u(k)) \leq \gamma \xi(k-1, x(k-1), u(k-1))$ is satisfied for all $k>0$. The closed-loop system is asymptotically attractive.

\section{Summary procedure 3}

\section{Offline:}

1. Choose $\tau$ and $\gamma$.

Online (at time step $k$ ):

Copyright (C) 2018 John Wiley \& Sons, Ltd. Received: 12 Dec 2017. Revised: 2019.Int. J. Robust. Nonlinear Control (2018), 
1. If $M_{k}$ does not exist, or does not satisfy (44), solve (48) subject to (49) and compute $M_{k}$ as defined by (43). Otherwise use existing values of $P_{k+1}, P_{k}, Q_{k}, R_{k}, S_{k}$ in (43) and current values of $x(k), y(k), f_{k}, G_{k}$ in (43).

2. Solve (30) subject to (46)

3. If infeasible, add small constant to right-hand side of (46) and repeat.

4. Set $u(k)=u_{0}$, where $\mathbf{u}=\left\{u_{0}, \ldots, u_{N}\right\}$ is the obtained solution.

\subsection{Application with persistent disturbances}

Sections 4.1-4.3 above demonstrated the use of Theorem 1 together with Lemma 1, with the assumption that there are no disturbances. Now we will assume that persistent disturbances are present (namely that $d(k) \neq 0,\|d(k)\|^{2} \leq \theta \neq 0$ in equation (1)) and demonstrate the use of Theorem 2. The key differences now are that inequalities (8) and (9) must be satisfied, rather than (4) and (5). This results in relatively small differences from the developments presented in sections 4.2 and 4.3 , as will now be detailed in the next two subsections.

4.4.1. MPC with linear system Assuming the linear model

$$
x(k+1)=A x(k)+B u(k)+d(k)
$$

and choosing $V(x)=x^{T} P x\left(P=P^{T}>0\right)$ and $\xi(x, u)=x^{T} Q x+2 x^{T} S u+u^{T} R u$, as in section 4.2 , inequality (8) becomes

$$
\left[\begin{array}{lll}
x(k)^{T} & u(k)^{T} & d(k)^{T}
\end{array}\right] M^{d}\left[\begin{array}{c}
x(k) \\
u(k) \\
d(k)
\end{array}\right] \leq 0
$$

where $M^{d}$ is given by

$$
M^{d}=\left[\begin{array}{ccc}
A^{T} P A-\tau P-Q & A^{T} P B-S & A^{T} P \\
B^{T} P A-S^{T} & B^{T} P B-R & B^{T} P \\
P A & P B & P-\sigma_{1} I
\end{array}\right]
$$

so (8) is satisfied for every $x(k), u(k), d(k)$ if

$$
M^{d} \leq 0
$$

Note that $P-\sigma_{1} I \leq 0$ is a necessary condition for this to hold, so $\sigma_{1}$ has some minimum possible value $\left(\sigma_{\min }=\lambda_{\max }(P)\right)$ which depends on the choice of $P$. Inequality (53) is again an LMI in the variables $P, Q, R, S, \sigma_{1}$.

We can satisfy (9) by imposing the condition (34) and

$$
\xi(x(k), u(k)) \leq \gamma \xi(x(k-1), u(k-1))+\epsilon \theta
$$

Copyright (C) 2018 John Wiley \& Sons, Ltd. Received: 12 Dec 2017. Revised: 2019.Int. J. Robust. Nonlinear Control (2018), 
(which gives $0<\xi(x(k), u(k))<\gamma^{k} \xi(x(0), u(0))+\epsilon \theta /(1-\gamma)$ and we can take $\alpha(s, k)=\gamma^{k} s$ as the $\mathcal{K} \mathcal{L}$ function in (9)). This translates into the constraint

$$
\begin{aligned}
& u(k)^{T} R u(k)+2 u(k)^{T} S x(k) \leq \\
& \gamma\left[x(k-1)^{T}, u(k-1)^{T}\right]\left[\begin{array}{cc}
Q & S \\
S^{T} & R
\end{array}\right]\left[\begin{array}{c}
x(k-1) \\
u(k-1)
\end{array}\right] \\
& -x(k)^{T} Q x(k)+\epsilon \theta
\end{aligned}
$$

The addition of the term $\epsilon \theta$ in (55) is in effect the same as the heuristic modification of (33) which was mentioned at the end of section 4.2, though here it is added with a different motivation, and permanently rather than temporarily.

We remark that the infeasibility mentioned there is less likely to occur when a disturbance is present, because $x(k)$ can be significantly different from $x(k-1)$ even if $u(k-1) \approx 0$. Note that the choice of $\epsilon$ impacts the performance of the closed-loop system; as $\epsilon$ is increased then the set to which $x(k)$ converges increases. This can be seen from Theorem 2 and its proof, in particular from (84) in Appendix B.

The following proposition and procedure summarize the development in this sub-section:

\section{Proposition 4}

Consider the system (50) with constraint sets $\mathbb{X}$ and $\mathbb{U}$, the MPC problem (30), and some $0<\tau<1$, $0<\gamma<1$, and $\epsilon>0$. Let $P \succ 0, Q, R, S$, and $\sigma_{1}$ be a solution to (53). Suppose that the right hand side of (55) is positive for $x(k), x(k-1) \in \mathbb{X} \backslash\{0\}$ and $u(k-1) \in \mathbb{U} \backslash\{0\}$, for all $k>0$. Let the control sequence $\mathbf{u}(x(k))=\left\{u_{0}, \ldots, u_{N}\right\}$ be a solution to the MPC optimization (30) subject to (55) at time step $k>0$; Then the state feedback system (15) with $u(x(k))=u_{0} \in \mathbf{u}(x(k))$ is dissipative with $V(x)=x^{T} P x$ and $\xi(x, u)=x^{T} Q x+2 x^{T} S u+u^{T} R u+\sigma_{1} d^{T} d$, and the QDC $0 \leq \xi(x(k), u(k)) \leq \gamma \xi(x(k-1), u(k-1))+\epsilon \theta$ is satisfied for all $k>0$. The state vector of the closed loop converges to a ball centred on 0 whose radius is a $\mathcal{K}$-function of $\epsilon \theta$.

\section{Summary procedure 4}

\section{Offline:}

1. Estimate $\theta$ from knowledge of the application problem. Choose $\epsilon$ from performance requirements. Choose $\tau$ and $\gamma$.

2. Solve (53) for $P, Q, R, S, \sigma_{1}$ (see (52)).

\section{Online:}

1. Solve (30) subject to (55).

2. If infeasible, increase $\epsilon$ (or $\theta)$ and repeat.

3. Set $u(k)=u_{0}$, where $\mathbf{u}=\left\{u_{0}, \ldots, u_{N}\right\}$ is the obtained solution.

4.4.2. MPC with input-affine system We now consider the model

$$
x(k+1)=f(x(k))+G(x(k)) u(k)+d(k)
$$


and, as in section 4.3 we will write $f_{k}=f(x(k))$ and $G_{k}=G(x(k))$. Again we take $V(k, x)=$ $x^{T} P_{k} x$ for some $P_{k}=P_{k}^{T}>0$, define a 'virtual output' $y(k)=\left[x(k)^{T}, f_{k}^{T}\right]^{T}$ and define the supply function

$$
\xi(k, x(k), u(k))=y(k)^{T} Q_{k} y(k)+2 y(k)^{T} S_{k} u(k)+u(k)^{T} R_{k} u(k)
$$

Using the system model (56), condition (8) becomes

$$
\left[\begin{array}{lll}
u(k)^{T} & 1 & d(k)^{T}
\end{array}\right] M_{k}^{d}\left[\begin{array}{c}
u(k) \\
1 \\
d(k)
\end{array}\right] \leq 0
$$

where

$$
M_{k}^{d}=\left[\begin{array}{ccc}
G_{k}^{T} P_{k+1} G_{k}-R_{k} & G_{k}^{T} P_{k+1} f_{k}-S_{k}^{T} y(k) & G_{k}^{T} P_{k+1} \\
f_{k}^{T} P_{k+1} G_{k}-y(k)^{T} S_{k} & f_{k}^{T} P_{k+1} f_{k}-\tau x(k)^{T} P_{k} x(k)-y(k)^{T} Q_{k} y(k) & f_{k}^{T} P_{k+1} \\
P_{k+1} G_{k} & P_{k+1} f_{k} & P_{k+1}-\sigma_{1} I
\end{array}\right]
$$

and (58) is satisfied for all $u(k)$ and $d(k)$ if

$$
M_{k}^{d} \leq 0
$$

which is an LMI in $P_{k+1}, P_{k}, Q_{k}, R_{k}, S_{k}, \sigma_{1}$, or in $P_{k+1}, Q_{k}, R_{k}, S_{k}, \sigma_{1}$ if $P_{k}$ is taken to be the value found at the previous time step. (But note that $\sigma_{1}$ should be kept fixed, not allowed to vary with $k$.)

As in section 4.4.1, to satisfy (9) we impose condition (47) and

$$
\begin{aligned}
& u(k)^{T} R_{k} u(k)+2 u(k)^{T} S_{k} x(k) \leq \\
& \gamma\left[y(k-1)^{T}, u(k-1)^{T}\right]\left[\begin{array}{cc}
Q_{k} & S_{k} \\
S_{k}^{T} & R_{k}
\end{array}\right]\left[\begin{array}{c}
y(k-1) \\
u(k-1)
\end{array}\right] \\
&-y(k)^{T} Q_{k} y(k)+\epsilon \theta
\end{aligned}
$$

as constraints. As in section 4.3 we also need to add the constraints (49).

The following proposition and procedure summarize the development in this sub-section:

\section{Proposition 5}

Consider the system (56) with constraint sets $\mathbb{X}$ and $\mathbb{U}$, the MPC problem (30), and some $0<\tau<1$, $0<\gamma<1$, and $\epsilon>0$. Let $P_{k+1} \succ 0, P_{k} \succ 0, Q_{k}, R_{k}, S_{k}$, and $\sigma_{1}$ be a solution to (48) subject to (60) and (49), with the current values of $x(k), y(k), f_{k}, G_{k}$, at time step $k$. Suppose that the right hand side of (46) is positive for $x(k), x(k-1) \in \mathbb{X} \backslash\{0\}$ and $u(k-1) \in \mathbb{U} \backslash\{0\}$, for all $k>0$, where the values of $Q_{k-1}, R_{k-1}, S_{k-1}$ are taken as the values obtained for $Q_{k}, R_{k}, S_{k}$ at the previous time step. Let the control sequence $\mathbf{u}(x(k))=\left\{u_{0}, \ldots, u_{N}\right\}$ be a solution to the MPC optimization (30) subject to (61) at time step $k>0$. Then the state feedback system (56) with $u(x(k))=u_{0} \in \mathbf{u}(x(k))$ 
is dissipative with $V(k, x)=x^{T} P_{k} x$ and $\xi(k, x, u)=y T Q_{k} y+2 y^{T} S_{k} u+u^{T} R_{k} u+\sigma_{1} d^{T} d$, and the (time-dependent) QDC $0 \leq \xi(k, x(k), u(k)) \leq \gamma \xi(k-1, x(k-1), u(k-1))+\epsilon \theta$ is satisfied for all $k>0$. The state vector of the closed loop converges to a ball centred on 0 whose radius is a $\mathcal{K}$-function of $\epsilon \theta$.

\section{Summary procedure 5}

\section{Offline:}

1. Estimate $\theta$ from knowledge of the application problem. Choose $\epsilon$ from performance requirements. Choose $\tau$ and $\gamma$.

Online (at time step $k$ ):

1. If $M_{k}^{d}$ does not exist, or does not satisfy (60), solve (48) subject to (60) (instead of (44)) and (49), computing $M_{k}^{d}$ as defined by (59). Otherwise use existing values of $P_{k+1}, P_{k}, Q_{k}, R_{k}, S_{k}$ in (59) and current values of $x(k), y(k), f_{k}, G_{k}$ in (59). Keep $\sigma_{1}$ fixed for all $k$ (for example, as the value obtained from the first solution of (48).)

2. Solve (30) subject to (61).

3. If infeasible, increase $\epsilon$ (or $\theta$ ) and repeat.

4. Set $u(k)=u_{0}$, where $\mathbf{u}=\left\{u_{0}, \ldots, u_{N}\right\}$ is the obtained solution.

\section{DISCUSSION}

\subsection{Flexibility in applications}

The theorems presented in section 3 are very general. The ways of exploiting them presented in section 4 are only examples. Some alternative ways of exploiting the theorems are readily apparent from the developments in sections 4.1 - 4.4. For example, in section 4.2 constraint (31) could be imposed online, using the measured value of $x(k)$, giving a less conservative solution at the expense of greater online computational complexity. Another rather obvious example is that, in all the cases where LMI's have been used (that is, (26)-(27), (35), (44), (53), (60)) it is possible to make various choices of which matrices to fix in advance and which should be variables in the LMI. The appropriate choice will vary from application to application and needs to be determined from simulation studies before implementation. Other less obvious variations are undoubtedly possible. Note that we have used quadratic storage and supply functions in section 4 because familiar LMI machinery then becomes available. But the theorems in section 3 do not require quadratic functions to be used.

\subsection{Relations to stability}

The theorems in section 3 establish convergence of the state to a point (Theorem 1) or to a bounded set (Theorem 2) from a particular initial condition. We wish to relate them to statements about stability of the system. For the simple case treated in section 4.1 (linear system with fixed state 
feedback and no disturbance) there is no issue, since satisfaction of (25) implies satisfaction of (23), and hence strict reduction of $\|x\|_{M}$ at each step, with $M>0$.

In the cases treated in sections 4.2-4.4 any conclusions about stability require the assumption that all constraints are feasible and satisfied at each $k$. This assumption is commonly made in stability proofs relating to MPC $[8,14,18]$, although it can be reduced to the assumption that the constraints are feasible and satisfied at some $k$ by adding 'recursive feasibility' constraints to the MPC problem [18]. In particular, imposing a terminal constraint set that is control invariant ensures recursive feasibility [1]. In principle, such a constraint could be added in the GDC setting, but as usual there may be difficulties in computing a suitable terminal constraint set, and there is an increased risk that the constraints are not feasible at any $k$. Furthermore, recall that in section 4.2 we introduced the GDC condition as an alternative to a terminal constraint set, in order to reduce the risk of initial infeasibility (ie in order to increase the domain of attraction). We therefore take the view that the GDC approach should normally be used without the imposition of recursive feasibility constraints. In practice this is not such a great loss, as most practical implementations of MPC systems do not impose recursive feasibility constraints, but rely instead on heuristics such as the use of softened constraints and choosing a long prediction horizon - note that we impose the GDC constraint only at the beginning of the horizon.

Most treatments of closed-loop stability of feedback systems that are based on notions of dissipativity proceed by examining two subsystems, establishing (or assuming) passivity of one and dissipativity of the other, and then inferring stability of the feedback connection of the two. For example, [5, 17] proceed in this way. By contrast, we consider the single closed-loop system, without examining its subsystems. Our conditions impose dissipation 'along trajectories', in a manner similar to that in [23, 12]. From a 'behavioural' point of view [16], in which a system is viewed as the set of possible trajectories of signals at its interfaces, our dissipativity constraints, together with the system being controlled and the MPC algorithm, in fact define a new (closedloop) system. This system has some stability properties by construction, in the sense that its state converges to either a point or a set, depending on whether persistent disturbances are present or not.

What kind of stability do we achieve? We certainly achieve Lagrange stability [11], that is the property that the state trajectory remains bounded, since that is explicitly proved in both Theorems 1 and 2. We also achieve convergence, as stated above. We do not, however, guarantee Lyapunov stability, because we do not guarantee continuity of the trajectory with respect to the initial condition, when it is perturbed from the equilibrium. This is precisely what is lost by allowing the storage function $V$ to increase within bounds. (Note that it is possible to obtain Lyapunov stability while allowing $V$ to increase, as in [31], but a sufficiently high overall 'rate of decrease' has to be specified.)

While this appears to be a bad situation, it is mitigated in practice because the continuity property is usually obtained as a result of the initial MPC problem itself (that is, by the cost function and constraints defined in (30)). So a well-founded MPC problem definition gives us reasonable shortterm behaviour (as one might expect from the solution of an open-loop problem, as in (30)), while the GDC condition ensures reasonable long-term behaviour. If both Theorem 2 and Corollary 1 hold, then (13) holds, which defines input-to-state stability [22, 7]. We enforce Theorem 2 by means of constraints added to the MPC algorithm, but Corollary 1 does not necessarily hold. If we use 
quadratic supply functions as defined in (16) or (40) then

$$
\xi(0, x(0), u(0))=\left[x(0)^{T}, u(0)^{T}\right]\left[\begin{array}{ll}
Q_{0} & S_{0} \\
S_{0}^{T} & R_{0}
\end{array}\right]\left[\begin{array}{l}
x(0) \\
u(0)
\end{array}\right]
$$

so that $|\xi(0, x(0), u(0))| \leq\left\|\left[x(0)^{T}, u(0)^{T}\right]\right\|^{2} \lambda_{\max }$, where $\lambda_{\max }$ denotes the largest eigenvalue of the matrix which appears in (62). Note that $\lambda_{\max }$ is a constant when the parameters of $\xi$ are fixed, and $\lambda_{\max } \leq c_{2}$ when the parameters of $\xi$ vary (see (49)). So Corollary 1 would hold if $\|u(0)\|$ were bounded by a $\mathcal{K}$-function of $\|x(0)\|$. In particular, Corollary 1 holds whenever $u(0)$ is a continuous function of $x(0)$ (see Remark 3) - and hence, whenever the control law is continuous in the state — providing that $\xi$ is continuous in the state and the input.

\subsection{Novel features}

As mentioned in the Introduction, there are overlaps with previous work on MPC which applies constraints arising from dissipativity concepts. The main innovation in this paper is the General Dissipativity Constraint ((4) or (8)) with very general storage and supply functions, which need to satisfy weak conditions ((2), specialised later to (9) and (10)). Even in the specialisation to the Quadratic Dissipativity Constraint in section 4 the quadratic supply functions remain relatively general. We have developed procedures which minimise the online computational burden by shifting offline the solutions to LMIs whenever possible.

The supply functions $\xi$ that we allow are more general than those usually encountered in the existing literature on input-to-state stability, L2 gain estimation, etc. For example, the supply rate associated with an ISS Lyapunov function is of the form $-\beta_{1}(\|x\|)+\beta_{2}(\|u\|)$ [22]. In particular, "cross-terms", such as the product $x^{T} S u$ in (16), are allowed in our development. This facilitates finding an online solution to inequality constraints such as (33). A basic intuition here is that the input $u$ can be found in a direction which best drives $x$ towards 0 . In some sense the constraint can take advantage of the detailed interaction of the state and the input, reminiscent of the use of 'phase information' by [20]. Of course, supply functions with cross-terms have been widely used in systems theory - in particular the input-output product $u^{T} y$ is fundamental in passivity theory.

In $[9,10]$ the notion of " $\mathcal{K} L$ stability with respect to two measures" is defined as the property

$$
\omega_{1}(x(k)) \leq \alpha\left(\omega_{2}(x(0)), k\right)
$$

where $\omega_{1}$ and $\omega_{2}$ are continuous functions and $\alpha$ is a $\mathcal{K} L$-function. It is remarked that the choice $\omega_{1}(x)=\omega_{2}(x)=\|x\|$ corresponds to the definition of asymptotic stability of the origin. Our condition

$$
|\xi(k, x(k), u(k))| \leq \alpha(|\xi(0, x(0), u(0))|, k)
$$

that appears in Lemma 1 and in Theorem 2 is superficially similar to (63), but the function $\xi$ is allowed to depend on $k$ and $u$ as well as $x$. It is therefore significantly different, and does not imply any stability-like results until it is coupled with the GDC conditions (4) or (8). 


\section{EXAMPLE}

We consider the 2-input, 4-state linearized model of a helicopter presented by [4]. The model represents the longitudinal dynamics of the $\mathrm{CH}-47$ tandem-rotor helicopter in horizontal motion about a nominal air-speed of $40 \mathrm{~m} / \mathrm{s}$ (78 knots). The states are: horizontal velocity $V_{h}(\mathrm{~m} / \mathrm{s})$, vertical velocity $V_{z}(\mathrm{~m} / \mathrm{s})$, pitch rate $q$ (radians/s) and pitch angle $\theta$ (radians). The inputs are: collective rotor thrust $u_{1}$, and differential rotor thrust $u_{2}$. The continuous-time model is given by $\dot{x}=A_{c} x+B_{c} u$, with

$$
A_{c}=\left[\begin{array}{cccc}
-0.02 & 0.005 & 2.4 & -32 \\
-0.14 & 0.44 & -1.3 & -30 \\
0 & 0.018 & -1.6 & 1.2 \\
0 & 0 & 1 & 0
\end{array}\right], \quad B_{c}=\left[\begin{array}{cc}
0.14 & -0.12 \\
0.36 & -8.6 \\
0.35 & 0.009 \\
0 & 0
\end{array}\right]
$$

We assume here that all four states can be measured. This model is open-loop unstable with 3 unstable modes, the poles being $-2.2279,0.0652,0.4913 \pm 0.4151 i$. We choose $T_{s}=0.18 \mathrm{~s}$ as the sampling and update interval for an MPC controller. Discretising the model with this choice (assuming zero-order hold on the inputs and Euler integration over the update interval), gives a model of the form (15) with

$$
A=\left[\begin{array}{cccc}
0.9964 & 0.0011 & -0.0961 & -5.7449 \\
-0.0262 & 1.0815 & -0.6703 & -5.6010 \\
-3.83 \times 10^{-5} & 0.0030 & 0.7651 & 0.1807 \\
-2.33 \times 10^{-6} & 0.0003 & 0.1574 & 1.0173
\end{array}\right], \quad B=\left[\begin{array}{cc}
0.0274 & -0.0224 \\
0.0504 & -1.6107 \\
0.0552 & -0.0009 \\
0.0052 & -1.02 \times 10^{-5}
\end{array}\right]
$$

which has poles at $0.6696,1.0118,1.0894 \pm 0.0816 i$.

\subsection{Scenario 1: No disturbances}

We design an MPC controller with a prediction horizon $N=6$ steps (namely 1.08 seconds), and a quadratic stage cost $x(k)^{T} W_{x} x(k)+u(k)^{T} W_{u} u(k)$, with $W_{x}=\operatorname{diag}[1,1,1.5,1.5]$ and $W_{u}=$ $\operatorname{diag}[0.02,0.02]$ - so that the controller's objective is to regulate the state to $x=0$. We do not use a terminal cost.

In order to apply the QDC we need to find suitable matrices $P, Q, R, S$ for use in inequalities (33),(34),(35). Since the open-loop system is unstable, we do not attempt to satisfy (37), as that is impossible. We choose $P$ to be the solution to the discrete algebraic Riccati equation [14]

$$
P=A^{T} P A-\left(A^{T} P B\right)\left(W_{u}+B^{T} P B\right)^{-1}\left(B^{T} P A\right)+W_{x}
$$

so as to reduce the number of variables in the LMIs. This gives

$$
P=\left[\begin{array}{rrrr}
4.0794 & -0.0490 & -3.9108 & -36.471 \\
-0.0490 & 1.0097 & 0.0631 & 0.5551 \\
-3.9108 & 0.0631 & 11.785 & 66.230 \\
-36.471 & 0.5551 & 66.230 & 528.33
\end{array}\right]>0
$$

Copyright (C) 2018 John Wiley \& Sons, Ltd. Received: 12 Dec 2017. Revised: 2019.Int. J. Robust. Nonlinear Control (2018), 
Using this value of $P$ in (35), with $z=[6,4,-0.4,0.15]^{T}$ in (36) and $\tau=0.999$ gives

$$
\begin{gathered}
Q=\left[\begin{array}{rrrr}
0.3106 & -0.3662 & 0.0332 & -0.0299 \\
-0.3662 & 0.6157 & 0.0240 & -0.0127 \\
0.0332 & 0.0240 & 0.8750 & 0.0893 \\
-0.0299 & -0.0127 & 0.0893 & 1.2847
\end{array}\right] \times 10^{3} \\
R=\left[\begin{array}{rr}
859.8 & -0.0255 \\
-0.0255 & 861.1
\end{array}\right], \quad S=\left[\begin{array}{rr}
-0.1928 & -0.0384 \\
0.0228 & -0.8921 \\
1.0112 & 0.8387 \\
4.7298 & 6.0216
\end{array}\right]
\end{gathered}
$$

Note that (34) holds. We then solve the MPC problem at each step, with input constraints $\left|u_{i}(k)\right| \leq 1.1(i=1,2)$ and the constraint (33) with $\gamma=0.995$, starting from the initial condition $x(0)=[6,13,-0.42,0.18]^{T}$.

As the simulation progresses it sometimes happens that the problem mentioned at the end of section 4.2 occurs, namely that inequality (33) becomes infeasible, or close to being infeasible. The optimisation problem then becomes infeasible or ill-conditioned, and the result is very dependent on the implementation of the optimisation algorithm. ${ }^{\dagger}$ To overcome this we adopt the heuristic measure of adding a positive constant to the right-hand side of (33) when the constraint is reported to be infeasible by the optimisation algorithm. We have found 500 to be a suitable constant in this case. Note that the largest and smallest eigenvalues of $\left[\begin{array}{cc}Q & S \\ S^{T} & R\end{array}\right]$ are 1304 and 63, respectively, so that a change of 500 in $\xi$ corresponds to a change no larger than 2.8 in $\left\|\left[x^{T}, u^{T}\right]\right\|$.

The resulting state trajectories are shown in fig.1, starting from $x(0)=[6,13,-0.42,0.18]^{T}$, and the input trajectories are shown in fig.2. (Note that the pitch rate and angle have been plotted in degrees/second and degrees, respectively, to show more detail.) Figures 3 and 4 show the evolution of the supply function $\xi$ and of $\|x\|$, respectively. The simulations have been performed with the system evolving in continuous time, and the MPC (with QDC) operating at intervals of $T_{s}=0.18 \mathrm{~s}$, as can be seen from the piecewise-constant nature of the inputs and of the supply function $\xi$.

In fig. 3 it can be seen that the supply function stops decreasing from $t \approx 1.5 \mathrm{~s}$, and even increases occasionally, due to the heursitic modification of (33) described above. It then reduces again from $t \approx 11$ s onwards. Note from fig. 4 that $\|x\|$ does not reduce monotonically, as would be likely (but not necessary) with a conventional Lyapunov constraint. There is only a reducing upper bound on $\|x\|$, as can be seen from (73) or (86).

\subsection{Scenario 2: Persistent disturbance}

In this scenario we assume that the model is given by (50), with $A$ and $B$ as in (66), and that the continuous-time model (65) is subject to a persistent disturbance on the pitch acceleration, namely $d(t)=\left[0,0, d_{3}(t), 0\right]^{T}$, with

$$
d_{3}(t)=15 \sin (0.667 t+5) \sin (6.67 t)
$$

\footnotetext{
${ }_{\dagger}$ We have used SeDuMi version 1.3 called from Matlab R2016a on a 2.2GHz Intel Core i7 processor. 


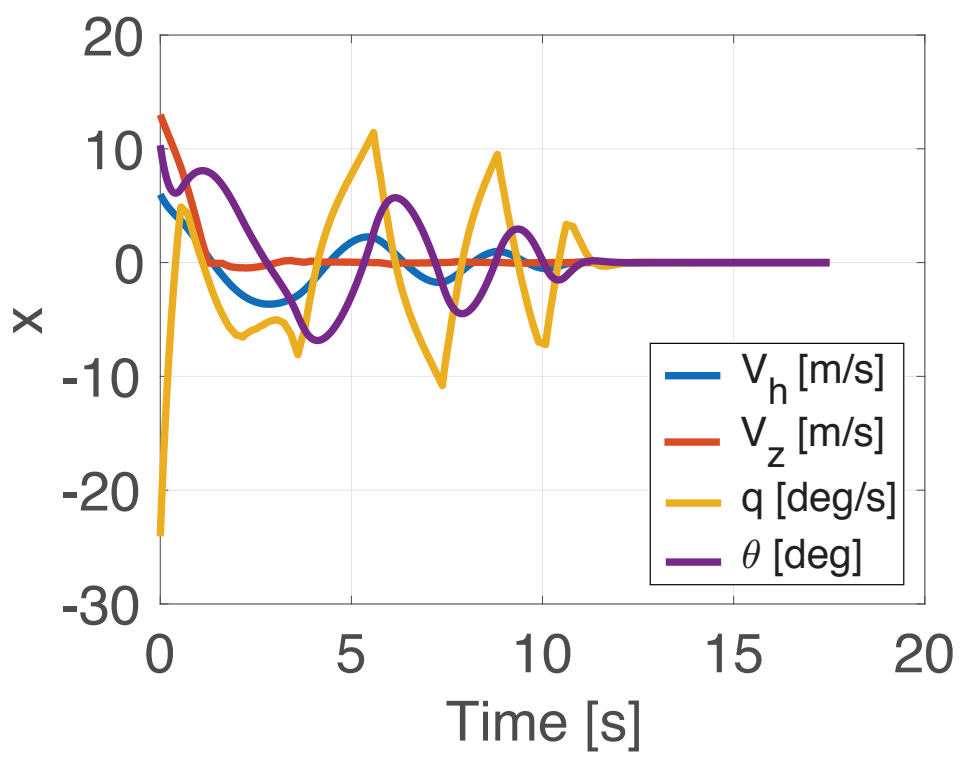

Figure 1: State trajectories with QDC, without disturbances.

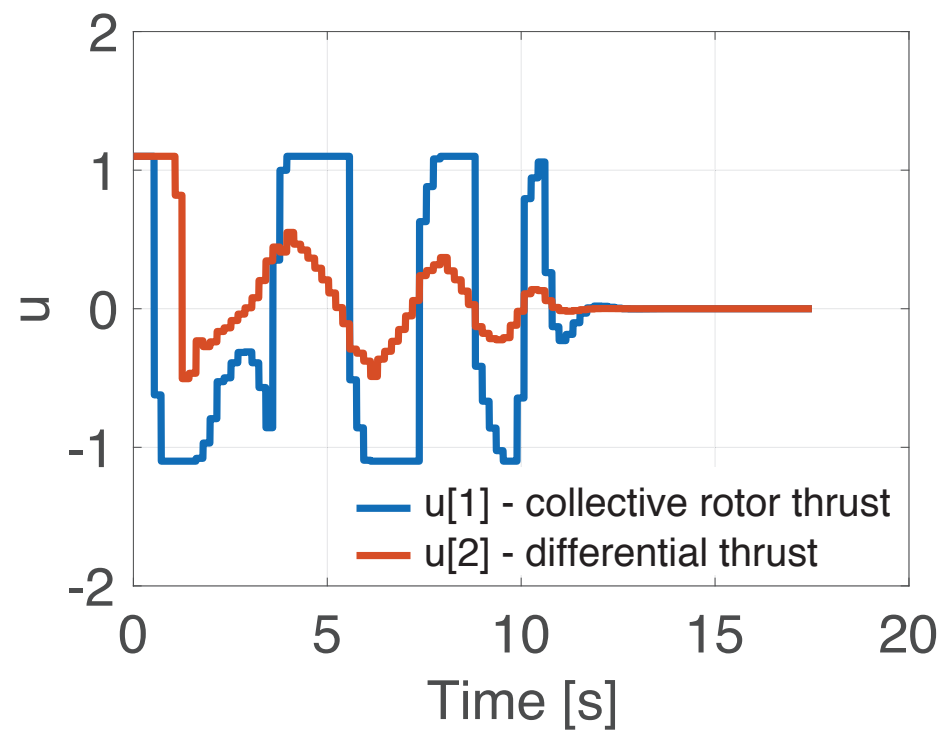

Figure 2: Input trajectories with QDC, without disturbances.

starting at $t=3.75 \mathrm{~s}$, as shown in Fig.5.

In this case the QDC condition becomes (54) (equivalent to (55)) and we choose (by off-line trial-and-error) $\epsilon \theta=300$. As was the case without disturbances, there is a possibility of this QDC condition becoming infeasible, and in case this happens we allow the possibility of increasing $\epsilon \theta$ to 350 .

State trajectories, starting from $x(0)=[6,13,-0.42,0.18]^{T}$ are shown in fig.6 (with the pitch rate and angle being plotted in degrees/second and degrees, respectively, as before). The input 


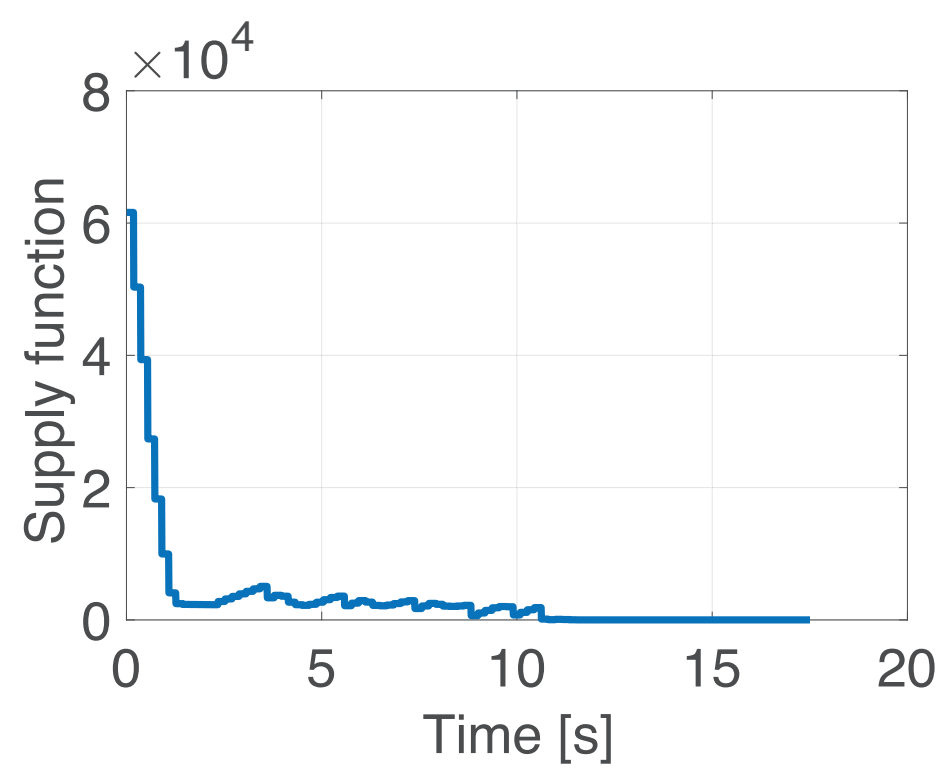

Figure 3: Evolution of the supply function $\xi$ with QDC, without disturbances.

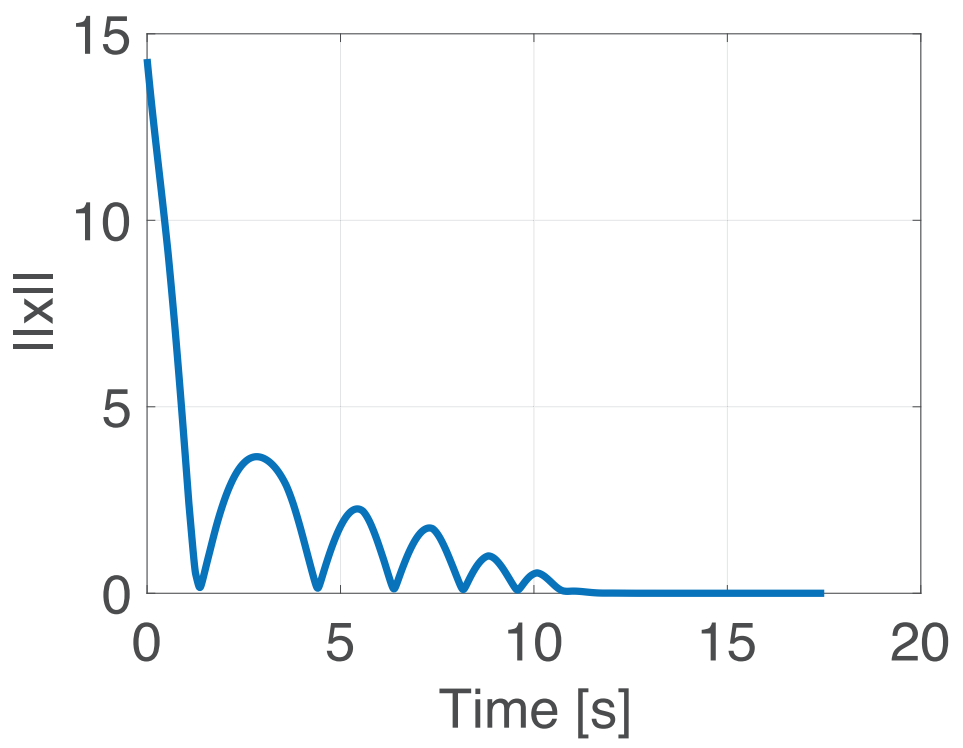

Figure 4: Evolution of $\|x\|$ with QDC, without disturbances.

trajectories are shown in fig.7. Figures 8 and 9 show the evolution of the supply function $\xi$ and of $\|x\|$, respectively. During this simulation $\epsilon \theta$ remains at 300, and is never increased to 350 .

As was the case without disturbances, there is an initial transient phase lasting about 12 seconds. This is followed by a steady-state phase in which the pitch rate oscillates with a peak value of about $1.5 \mathrm{deg} / \mathrm{s}$, and $\|x\|$ oscillates with a peak value of about 0.04 . Note that, with these MPC parameters, the closed loop is unstable if the QDC condition is not enforced. 


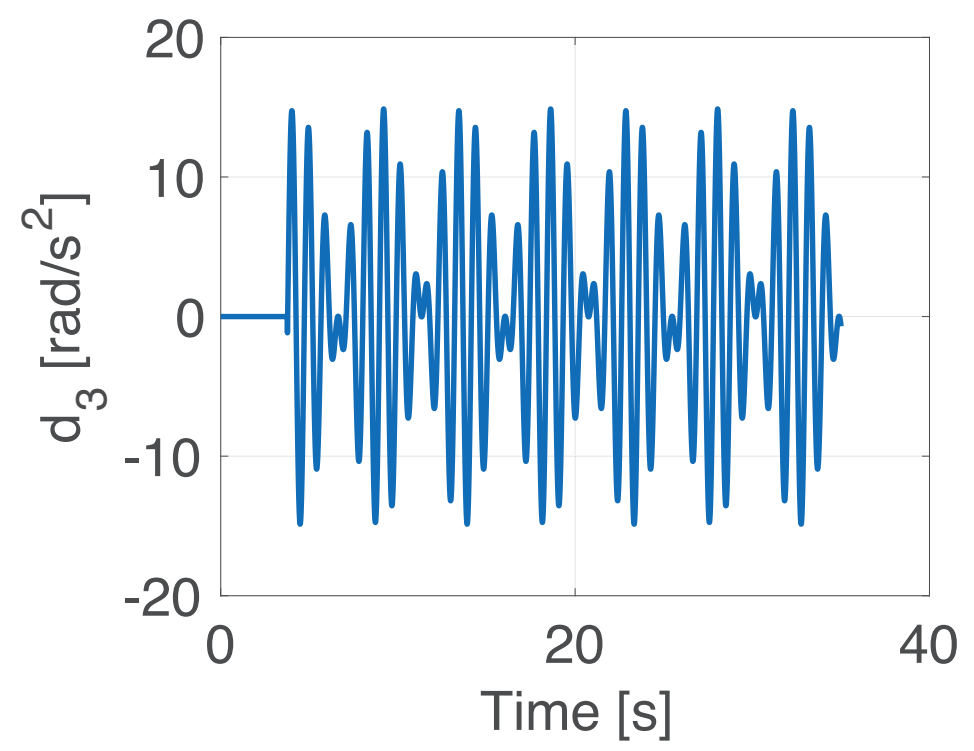

Figure 5: Disturbance on pitch acceleration

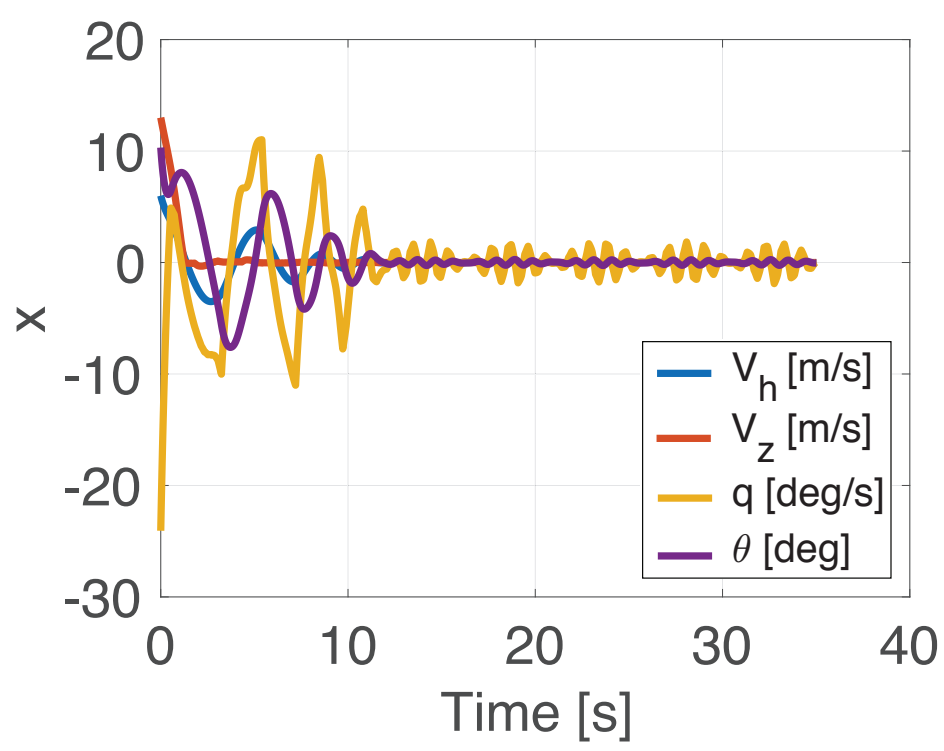

Figure 6: State trajectories with QDC, with disturbance.

\section{CONCLUSION}

We have introduced a 'general dissipativity constraint' (GDC) to assist the design of stable feedback systems, especially when MPC controllers are used and it is preferred to avoid the use of 'stabilising ingredients' such as terminal constraint sets or long prediction horizons. The stability that is guaranteed is weaker than Lyapunov stability, being 'Lagrange stability plus convergence'. This has little impact in practice, because the feature that is missing from Lyapunov stability usually 


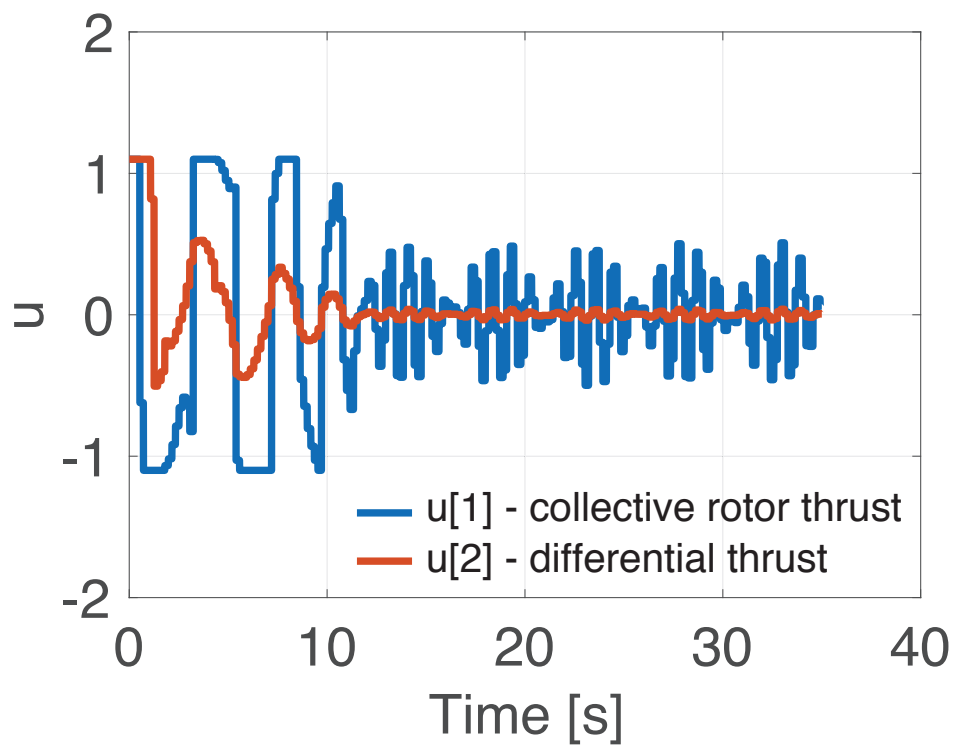

Figure 7: Input trajectories with QDC, with disturbance.

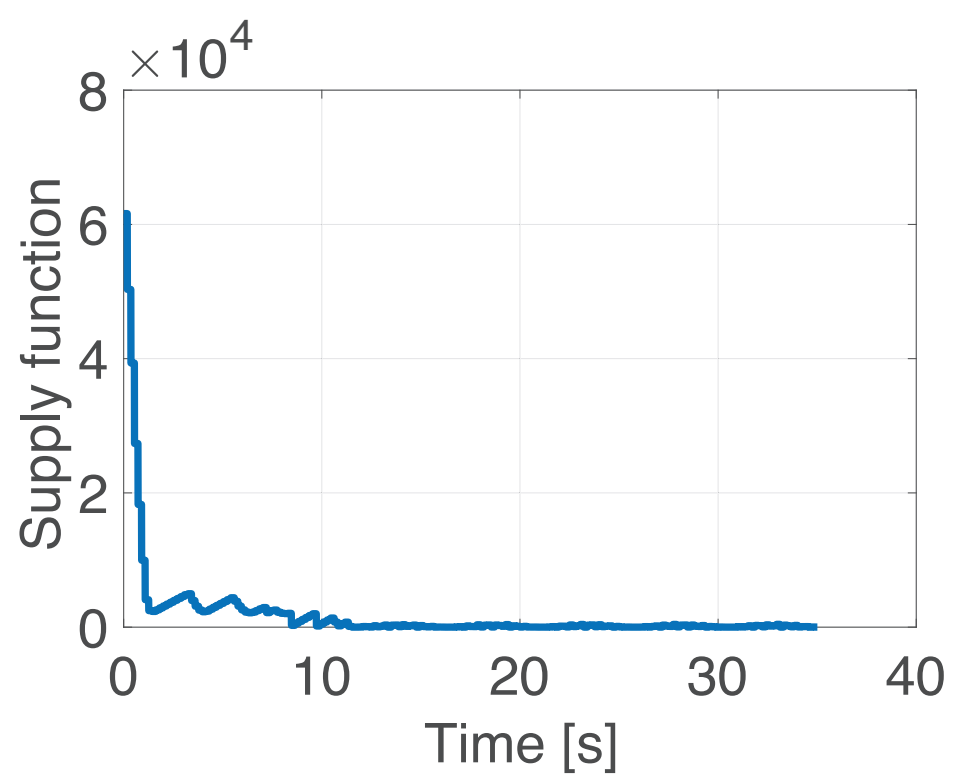

Figure 8: Evolution of the supply function $\xi$ with QDC, with disturbance.

arises from sensible MPC problem formulations. In particular, we have shown that we obtain inputto-state stability if the control law is continuous at 0 (at the set-point, in general). We have shown in detail how to apply the GDC concept in various scenarios, in each case specialising to a 'quadratic dissipativity constraint' (QDC) which allows implementation by the use of LMI techniques. An example has illustrated the efficacy of the approach in practice. Although section 6.1 showed the application of the theory when no disturbances are present, in practice one would always assume 


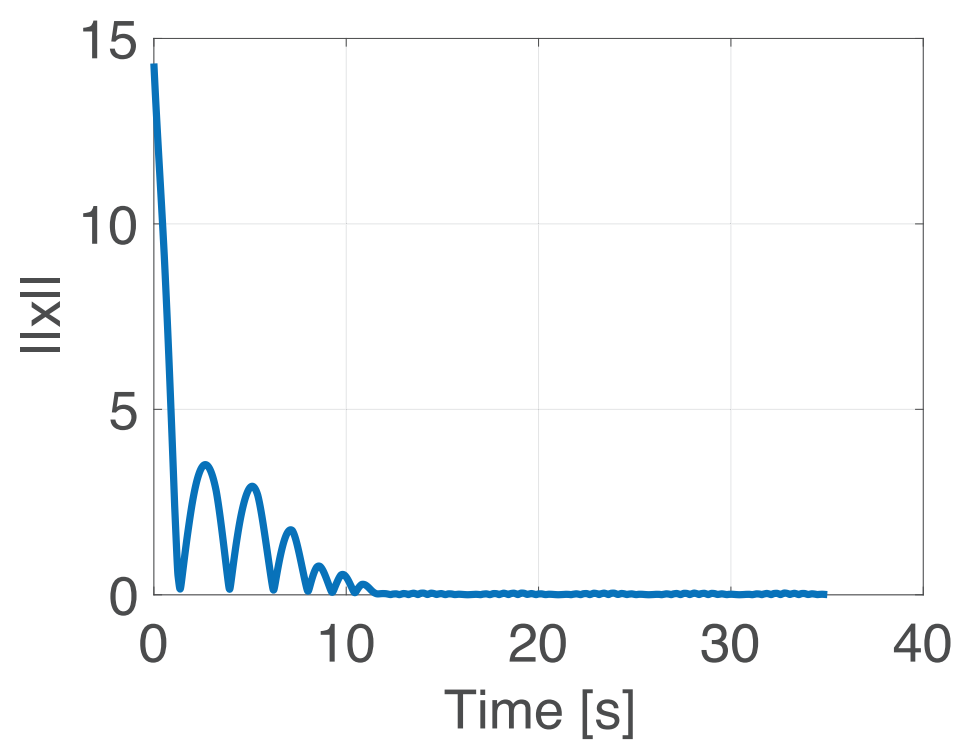

Figure 9: Evolution of $\|x\|$ with QDC, with disturbance.

the presence of some level of disturbance, so that section 6.2 is more representative of what would actually happen in a real application. The main utility of our approach is not that it can demonstrate existence of a stabilising control law, but that it can be used constructively to find such a law.

\section{REFERENCES}

1. F. Borrelli, A. Bemporad, and M. Morari. Predictive Control for Linear and Hybrid Systems. Cambridge University Press, 2017.

2. S. Boyd and L. Vandenberghe. Convex Optimization. Cambridge University Press, 2004.

3. H. Chen and C.W. Scherer. Moving horizon $H_{\infty}$ control with performance adaptation for constrained linear systems. Automatica, 42:1033-1040, 2006.

4. J.C. Doyle and G. Stein. Multivariable feedback design: Concepts for a classic/modern synthesis. IEEE Transactions on Automatic Control, 26(1):4-16, 1981.

5. D.L. Hill and P.J. Moylan. The stability of nonlinear dissipative systems. IEEE Transactions on Automatic Control, 21(3):708-711, 1976.

6. H.M. James, N.B. Nichols, and R.S. Philips. Theory of Servomechanisms. McGraw-Hill, 1947.

7. Z.P. Jiang and Y. Wang. Input-to-state stability for discrete-time nonlinear systems. Automatica, 37:875-869, 2001.

8. S.S. Keerthi and E.G. Gilbert. Optimal infinite-horizon feedback laws for a general class of constrained discretetime systems: Stability and moving horizon approximations. Journal of Optimization Theory and Applications, 57(2):265-29, 1988.

9. C.M. Kellet and A.R. Teel. On the robustness of KL-stability for difference inclusions: Smooth discrete-time Lyapunov functions. SIAM Journal on Control and Optimization, 44(3):777-800, 2005.

10. C.M. Kellett and A.R. Teel. Sufficient conditions for robustness of KL-stability for difference inclusions. Mathematics of Control Signals and Systems, 19:183-205, 2007.

11. H.K. Khalil. Nonlinear Systems. Pearson Education, 2002.

12. J.P. Koeln and A.G. Alleyne. Stability of decentralized model predictive control of graph-based power flow systems via passivity. Automatica, 82:29-34, 2017.

13. M. Lazar and R.H. Gielen. On parameterized dissipation inequalities and receding horizon robust control. International Journal of Robust and Nonlinear Control, 22(12):1314-1329, 2012. 
14. J.M. Maciejowski. Predictive Control with Constraints. Prentice Hall, 2002.

15. A.V. Oppenheim, A.S. Willsky, and I.T. Young. Signals and Systems. Prentice Hall, 1984.

16. J.W. Polderman and J.C. Willems. Introduction to Mathematical Systems Theory: A Behavioural Approach. Springer, 2007.

17. T. Raff, C. Ebenbauer, and F. Allgower. Nonlinear model predictive control: A passivity-based approach. In R. Findeisen, F. Allgower, and L. Biegler, editors, Assessment and Future Directions of Nonlinear Model Predictive Control, pages 151-162. Springer, 2007.

18. J.B. Rawlings and D.Q. Mayne. Model Predictive Control: Theory and Design. Nob Hill Publishing, Wisconsin, 2009.

19. C. Scherer, P. Gahinet, and M. Chilali. Multiobjective output-feedback control via LMI optimization. IEEE Transactions on Automatic Control, 42(7):896-911, 1997.

20. R. Sepulchre, M. Jankovic, and P.V. Kokotovic. Constructive Nonlinear Control. Springer, 1997.

21. E.D. Sontag. Smooth stabilization implies coprime factorization. IEEE Transactions on Automatic Control, 34:435-443, 1989.

22. E.D. Sontag and Y. Wang. On characterizations of the input-to-state stability property. Systems and Control Letters, 24:351-359, 1995.

23. M.J. Tippett and J. Bao. Distributed model predictive control based on dissipativity. AIChE Journal, 59(3):787804, 2013.

24. Tri Tran, E. Foo, K-V. Ling, and J.M. Maciejowski. Application of quadratically-constrained model predictive control in power systems. In Proc. $13^{\text {th }}$ International Conference on Automation, Robotic, Control and Vision, pages 193-198, Singapore, 122014.

25. Tri Tran and Q. P. Ha. Networked control systems with accumulative quadratic constraint. Electronics Letters, 4(2):108-110, 2011.

26. Tri Tran, K-V. Ling, and J.M. Maciejowski. Closed-loop development for dissipativity constraint. In Proc. $3^{\text {rd }}$ International Conference on Control, Automation, and Information Science, pages 24-29, Gwangyu, South Korea, 122014.

27. Tri Tran, K-V. Ling, and J.M. Maciejowski. Model predictive control of nonlinear input-affine systems with feasibility and stability constraints. In Proc. $13^{\text {th }}$ International Conference on Automation, Robotic, Control and Vision, pages 992-997, Singapore, 122014.

28. Tri Tran, K-V. Ling, and J.M. Maciejowski. Model predictive control via quadratic dissipativity constraint. In Proc. 53 ${ }^{\text {rd }}$ IEEE Conference on Decison and Control, pages 6689-6694, Los Angeles, 122014.

29. Tri Tran, D. Zhou, K-V. Ling, and J.M. Maciejowski. Multiplexed model predictive control of interconnected systems. In Proc. 54 ${ }^{\text {th }}$ IEEE Conference on Decision and Control, pages 2383-2388, Osaka, Japan, 122015.

30. J.C. Willems. Dissipative dynamical systems. European Journal of Control, 13:134-151, 2007.

31. Q. Yu and B. Yu. Generalized Lyapunov function theorems and its applications in switched systems. Systems and Control Letters, 77:40-45, 2015.

\section{A. PROOF OF THEOREM 1}

From (4) we get the following inequality for all $k>0$ :

$$
\begin{aligned}
V(k+1, x(k+1)) \leq & \tau V(k, x(k))+|\xi(k, x(k), u(k))| \\
\leq & \tau[\tau V(k-1, x(k-1))+|\xi(k-1, x(k-1), u(k-1))|] \\
& +|\xi(k, x(k), u(k))| \\
= & \tau^{2} V(k-1, x(k-1)) \\
& +[\tau|\xi(k-1, x(k-1), u(k-1))|+|\xi(k, x(k), u(k))|] .
\end{aligned}
$$

Copyright (C) 2018 John Wiley \& Sons, Ltd. Received: 12 Dec 2017. Revised: 2019.Int. J. Robust. Nonlinear Control (2018) 
Continuing in this way, we get

$$
V(k+1, x(k+1)) \leq \tau^{k+1} V(0, x(0))+\sum_{i=0}^{k} \tau^{i}|\xi(k-i, x(k-i), u(k-i))| .
$$

Note that the second term in (73) has the form of a convolution sum, so we can use a couple of results from linear systems theory:

Lemma 2

Consider two sequences $\left\{g_{k}\right\},\left\{h_{k}\right\}$, and assume that $\sum_{k=0}^{\infty}\left|g_{k}\right|=G<\infty$. Then

1. If $\sup _{k}\left|h_{k}\right|=H<\infty$ then $\left|\sum_{i=0}^{k} g_{i} h_{k-i}\right| \leq G H<\infty$ for all $k$.

2. If $\sum_{k=0}^{\infty}\left|h_{k}\right|=H<\infty$ then $\lim _{k \rightarrow \infty} \sum_{i=0}^{k} g_{i} h_{k-i}=0$,

Proof:

1. This is standard in linear systems theory $[6,15]$, but we give the proof here for completeness.

$$
\left|\sum_{i=0}^{k} g_{i} h_{k-i}\right| \leq \sum_{i=0}^{k}\left|g_{i}\right|\left|h_{k-i}\right| \leq H \sum_{i=0}^{k}\left|g_{i}\right| \leq H \sum_{i=0}^{\infty}\left|g_{i}\right|=G H
$$

2. This states that the output of an asymptotically stable linear system converges to 0 if the input converges to 0 sufficiently quickly to be absolutely summable. Although this seems almost self-evident, we have not found a proof in the literature, so we present one here. Since both $\left\{g_{k}\right\}$ and $\left\{h_{k}\right\}$ are absolutely summable there must exist $k_{1}$ and $k_{2}$ large enough that $\sum_{k=k_{1}}^{\infty}\left|g_{k}\right|<\epsilon$ and $\sum_{k=k_{2}}^{\infty}\left|h_{k}\right|<\epsilon$ for any $\epsilon>0$. Now consider $k>k_{1}+k_{2}-1$. Then

$$
\left|\sum_{i=0}^{k} g_{i} h_{k-i}\right| \leq\left|\sum_{i=0}^{k_{1}-1} g_{i} h_{k-i}\right|+\left|\sum_{i=k_{1}}^{k} g_{i} h_{k-i}\right|<\left|\sum_{j=k_{2}}^{k} g_{k-j} h_{j}\right|+\left|\sum_{i=k_{1}}^{k} g_{i} h_{k-i}\right|
$$

where we have made the substitution $j=k-i$ in the first sum on the right-hand side and re-ordered the terms in that sum. Now using the fact that $\sum_{k}\left|a_{k} b_{k}\right| \leq\left(\sum_{k}\left|a_{k}\right|\right)\left(\sum_{k}\left|b_{k}\right|\right)$, we see that the right-hand side can be no larger than $\epsilon(G+H)$. But $\epsilon$ can be chosen to be arbitrarily small, so $\left|\sum_{i=0}^{k} g_{i} h_{k-i}\right|$ can be made arbitrarily small by choosing $k$ large enough.

Applying Lemma 2 to the second term on the right hand side of (73) (taking $g_{k}=$ $|\xi(k, x(k), u(k))|$ and $h_{k}=\tau^{k}$ and recalling that (2) holds), we see that both terms on the right hand side of (73) remain bounded and converge to zero as $k \rightarrow \infty$. But $V(k, x(k)) \geq 0$ by assumption, so $V(k, x(k)) \rightarrow 0$ as $k \rightarrow \infty$. From (3) we deduce that $x(k)$ remains bounded and that $\|x(k)\| \rightarrow 0$ as $k \rightarrow \infty$.

\section{B. PROOF OF THEOREM 2}


From (8) the following inequality is obtained for all $k>0$ :

$$
\begin{aligned}
V(k+1, x(k+1)) \leq & \tau V(k, x(k))+|\xi(k, x(k), u(k))|+\sigma_{1} d(k)^{T} d(k) \\
\leq & \tau^{2} V(k-1, x(k-1)) \\
& +[\tau|\xi(k-1, x(k-1), u(k-1))|+|\xi(k, x(k), u(k))|] \\
& +\sigma_{1}\left[\tau d(k-1)^{T} d(k-1)+d(k)^{T} d(k)\right] .
\end{aligned}
$$

Continuing in this way, as in the proof of Theorem 1, using $d^{T}(k) d(k) \leq \theta$ for each $k$, and (9), we get

$$
\begin{aligned}
& V(k+1, x(k+1)) \leq \tau^{k+1} V(0, x(0))+ \\
& \sum_{i=0}^{k} \tau^{i}\left[\alpha(|\xi(0, x(0), u(0))|, k-i)+\sigma_{2} \theta\right]+\sigma_{1} \theta \sum_{i=0}^{k} \tau^{i} \\
& =\tau^{k+1} V(0, x(0))+\sum_{i=0}^{k} \tau^{i} \alpha(|\xi(0, x(0), u(0))|, k-i)+\left(\sigma_{1}+\sigma_{2}\right) \theta \sum_{i=0}^{k} \tau^{i}
\end{aligned}
$$

We can apply Lemma 2 to the second term in (76) by taking $g_{k}=\alpha(|\xi(0, x(0), u(0))|, k)$ and $h_{k}=\tau^{k}$. The assumed bounded-sum conditions in the Lemma hold for $g_{k}$ from (6) and for $h_{k}$ since $0<\tau<1$.

If $\tau \neq \gamma$ let

$$
M(k)=\gamma^{k} \frac{1-\left(\frac{\tau}{\gamma}\right)^{k}}{1-\left(\frac{\tau}{\gamma}\right)}=\tau^{k} \frac{1-\left(\frac{\gamma}{\tau}\right)^{k}}{1-\left(\frac{\gamma}{\tau}\right)}
$$

Then the second term in (76) can be bounded as follows:

$$
\begin{aligned}
\sum_{i=0}^{k} \tau^{i} \alpha(|\xi(0, x(0), u(0))|, k-i) & \leq \alpha(|\xi(0, x(0), u(0))|, 0) \sum_{i=0}^{k} \tau^{i} \gamma^{k-i} \\
& =\alpha(|\xi(0, x(0), u(0))|, 0) M(k)
\end{aligned}
$$

If $\tau<\gamma$ we have $M(k)<\frac{\gamma^{k}}{1-\left(\frac{\tau}{\gamma}\right)}$ and if $\tau>\gamma$ we have $M(k)<\frac{\tau^{k}}{1-\left(\frac{\gamma}{\tau}\right)}$. If $\tau=\gamma$ then the sum in (78) becomes

$$
\sum_{i=0}^{k} \gamma^{k}=(k+1) \gamma^{k}
$$

which has a maximum at $k^{*}=-(1 / \ln \gamma)$ (considering $k$ to be a real variable for this purpose).

So we have

$$
\sum_{i=0}^{k} \tau^{i} \alpha(|\xi(0, x(0), u(0))|, k-i) \leq \alpha_{3}(|\xi(0, x(0), u(0))|, k)
$$


where

$$
\begin{aligned}
& \alpha_{3}(|\xi(0, x(0), u(0))|, k)= \\
& \qquad \begin{cases}\alpha(|\xi(0, x(0), u(0))|, 0) \frac{\gamma^{k}}{1-\left(\frac{\tau}{\gamma}\right)} & \text { if } \tau<\gamma \\
\alpha(|\xi(0, x(0), u(0))|, 0) \frac{\tau^{k}}{1-\left(\frac{\gamma}{\tau}\right)} & \text { if } \tau>\gamma \\
\left\{\begin{array}{ll}
\alpha(|\xi(0, x(0), u(0))|, 0)\left(1+k^{*}-k\right) k^{*} \gamma^{k^{*}} & \text { if } k \leq\left\lfloor k^{*}\right\rfloor \\
\alpha(|\xi(0, x(0), u(0))|, 0) k \gamma^{k} & \text { if } k>\left\lfloor k^{*}\right\rfloor
\end{array}\right\} & \text { if } \tau=\gamma\end{cases}
\end{aligned}
$$

is a $\mathcal{K} \mathcal{L}$-function. (Of course many other bounding $\mathcal{K} \mathcal{L}$-functions exist; this development illustrates that at least one suitable bound exists.)

Putting this together with (76) we get

$$
V(k+1, x(k+1)) \leq \tau^{k+1} V(0, x(0))+\alpha_{3}(|\xi(0, x(0), u(0))|, k)+\frac{\left(\sigma_{1}+\sigma_{2}\right) \theta}{1-\tau} .
$$

So now we have a bound on $V$. To complete the proof we need a bound on $\|x\|$, as in (11). This is easily done using (7) from which we infer that $\|x(k)\| \leq \underline{\alpha}^{-1}(V(k, x(k)))$; note that $\underline{\alpha}^{-1}(\cdot)$ is a $\mathcal{K}$-function. Now we use the facts that the composition of $\mathcal{K}$-functions is a $\mathcal{K}$-function, and that for any $\mathcal{K}$-function $\alpha, \alpha(x+y+z) \leq \alpha(2 x)+\alpha(4 y)+\alpha(4 z)$ (which is a consequence of the known result that $\alpha(x+y) \leq \alpha(2 x)+\alpha(2 y)[21])$ :

$$
\begin{aligned}
\|x(k)\| \leq & \underline{\alpha}^{-1}\left(\tau^{k} V(0, x(0))+\alpha_{3}(|\xi(0, x(0), u(0))|, k-1)+\frac{\left(\sigma_{1}+\sigma_{2}\right) \theta}{1-\tau}\right) \\
\leq & \underline{\alpha}^{-1}\left(2 \tau^{k} V(0, x(0))\right)+\underline{\alpha}^{-1}\left(4 \alpha_{3}(|\xi(0, x(0), u(0))|, k-1)\right)+ \\
& +\underline{\alpha}^{-1}\left(4 \frac{\left(\sigma_{1}+\sigma_{2}\right) \theta}{1-\tau}\right) \\
\triangleq & \alpha_{0}(\|x(0)\|, k)+\alpha_{1}(|\xi(0, x(0), u(0))|, k)+\beta(\theta)
\end{aligned}
$$

where $\alpha_{0}$ and $\alpha_{1}$ are $\mathcal{K} \mathcal{L}$-functions and $\beta$ is a $\mathcal{K}$-function. $\alpha_{0}$ is defined as:

$$
\alpha_{0}(\|x(0)\|, k)=\underline{\alpha}^{-1}\left(2 \tau^{k} \bar{\alpha}(\|x(0)\|)\right)
$$

and the definitions of $\alpha_{1}$ and $\beta$ are obvious. 